\title{
Asymptotic Expansion of Singular Solutions and the Characteristic Polygon of Linear Partial Differential Equations in the Complex Domain
}

\author{
Dedicated to Professor Kunihiko Kajitani on his sixtieth birthday
}

By

Sunao ŌUCHI*

\begin{abstract}
Let $P(z, \partial)$ be a linear partial differential operator with holomorphic coefficients in a neighborhood $\Omega$ of $z=0$ in $\mathbb{C}^{d+1}$. Consider the equation $P(z, \partial) u(z)=f(z)$, where $u(z)$ admits singularities on the surface $K=\left\{z_{0}=0\right\}$ and $f(z)$ has an asymptotic expansion of Gevrey type with respect to $z_{0}$ as $z_{0} \rightarrow 0$. We study the possibility of asymptotic expansion of $u(z)$. We define the characteristic polygon of $P(z, \partial)$ with respect to $K$ and characteristic indices. We discuss the behavior of $u(z)$ in a neighborhood of $K$, by using these notions. The main result is a generalization of that in [6].

KEY WORDS: complex partial differential equations, solutions with asymptotic expansion
\end{abstract}

\section{$\S 0$. Introduction}

Let $P(z, \partial)$ be a linear partial differential operator with order $m$ and holomorphic coefficients in a domain containing the origin $z=0$ in $\mathbb{C}^{d+1}$ and $K=\left\{z_{0}=0\right\}$. In the present paper we consider

$$
P(z, \partial) u(z)=f(z)
$$

where $u(z)$ and $f(z)$ are holomorphic except on $K$. The purpose of the present paper is to study the behavior of singular solutions near $K$. First we note that for given $P(z, \partial)$ we can define the characteristic polygon $\Sigma$ with respect to $K$

Communicated by T. Kawai, August 26, 1999. Revised February 28, 2000.

1991 Mathematics Subject Classification(s): Primary 35A20; Secondary 35B40, 35C20.

* Department of Mathematics, Sophia University, Chiyoda-ku, Tokyo 102-8554, Japan.

e-mail: s_ohuti@hoffman.cc.sophia.ac.jp 
and exponents $\gamma_{\imath}(0 \leq i \leq p), 0=\gamma_{p}<\gamma_{p-1}<\cdots<\gamma_{1}<\gamma_{0}=+\infty$, which are the slopes of the characteristic polygon $\Sigma$ and called characteristic indices with respect to $K$. We study the relations between behaviors of singular solutions near $K$ and the characteristic indices.

Roughly speaking, the main result is the following:

Consider (Eq) and suppose that the following conditions (1), (2) and (3) are satisfied.

(1) $u(z)$ grows at most in some exponential order near $z_{0}=0$, that is, there is $a \gamma>0$ such that for any $\varepsilon>0|u(z)| \leq C_{\varepsilon} \exp \left(\varepsilon\left|z_{0}\right|^{-\gamma}\right)$.

(2) $f(z)$ has a Gevrey type asymptotic expansion, $f(z) \sim \sum_{n=0}^{+\infty} f_{n}\left(z^{\prime}\right) z_{0}^{n}$ with $\left|f_{n}\left(z^{\prime}\right)\right| \leq A B^{n} \Gamma(n / \gamma+1)$, as $z_{0} \rightarrow 0$ in a sectorial region $\Omega(\theta)$ with respect to $\approx 0$.

(3) The traces of $u(z)$ on some hypersurface $S$ have also the asymptotic expansion of the same type as $f(z)$.

Then we conclude under some conditions on $P(z, \partial), \gamma$ and $S$ that $u(z)$ has also an asymptotic expansion of the same Gevrey type as $f(z)$, as $z_{0}$ tends to 0 .

In the above assertion $\gamma$ is one of $\gamma_{2}(i=0,1, \cdots, p-1)$. It is a generalization of the main results of [5] and [6]. We treated the case $\gamma=\gamma_{p-1}$ in those papers. We studied in [5] the behavior of $u(z)$ by analysis of an integral representation of solutions with singularities on $K$. In [6] we did not use the representation but showed the possibility of asymptotic expansion of $u(z)$, by estimating the derivatives $\left(\partial / \partial z_{0}\right)^{n} u(z)$. So the arguments in [6] were less complicated and completely different from [5]. In the present paper we show the main result by the estimate of derivatives of $u(z)$, which follows [6].

In $\S 1$ we first define the characteristic polygon $\Sigma$. From $\Sigma$ we determine the indices $\gamma_{\imath}$ and polynomials $\chi_{P_{,}}\left(z^{\prime}, \xi^{\prime}\right)(0 \leq i \leq p-1)$. Next we introduce function spaces $\mathcal{O}(\Omega(\theta))$ and $A s y_{\{\kappa\}}(\Omega(\theta))$ which are subspaces of holomorphic functions except on $K$. We give the main results (Theorem 1.4) and examples. The proof is given in the following sections. In $\S 2$ we give majorant functions and estimate the derivatives of solutions. In $\S 3$, we give a result concerning functions with asymptotic expansion and by combining it with the estimate obtained, we complete the proof of Theorem 1.4.

\section{$\S 1 . \quad$ Notations and Results}

In this section we give notations and definitions and state results more precisely. The coordinates of $\mathbb{C}^{d+1}$ are denoted by $z=\left(z_{0}, z_{1}, \cdots, z_{d}\right)=\left(z_{0}, z^{\prime}\right) \in$ 
$\mathbb{C} \times \mathbb{C}^{d} .|z|=\max \left\{\left|z_{1}\right| ; 0 \leq i \leq d\right\}$ and $\left|z^{\prime}\right|=\max \left\{\left|z_{1}\right| ; 1 \leq i \leq d\right\}$. Its dual variables are $\xi=\left(\xi_{0}, \xi^{\prime}\right)=\left(\xi_{0}, \xi_{1}, \cdots, \xi_{d}\right)$. N is the set of all nonnegative integers, $\mathbb{N}=\{0,1,2, \cdots\}$. For real number $a,[a]$ means the integral part of $a$. The differentiation is denoted by $\partial_{\imath}=\partial / \partial z_{\imath}$, and $\partial=\left(\partial_{0}, \partial_{1}, \cdots, \partial_{d}\right)=\left(\partial_{0}, \partial^{\prime}\right)$. For a multi-index $\alpha=\left(\alpha_{0}, \alpha^{\prime}\right) \in \mathbb{N} \times \mathbb{N}^{d},|\alpha|=\alpha_{0}+\left|\alpha^{\prime}\right|=\sum_{\imath=0}^{d} \alpha_{\imath}$. Define $\partial^{\alpha}=\prod_{\imath=0}^{d} \partial_{\imath}^{\alpha_{\imath}}$. We denote $\partial^{\prime \alpha^{\prime}}=\prod_{l=1}^{d} \partial_{\imath}^{\alpha_{\imath}}$ by $\partial^{\alpha^{\prime}}$ and $\xi^{\prime \alpha^{\prime}}=\prod_{l=1}^{d} \xi_{\imath}^{\alpha_{\imath}}$ by $\xi^{\alpha^{\prime}}$.

Now let $P(z, \partial)$ be an $m$-th order linear partial differential operator with holomorphic coefficients in a neighborhood of $z=0$,

$$
P(z, \partial)=\sum_{|\alpha| \leq m} a_{\alpha}(z) \partial^{\alpha}
$$

Let $j_{\alpha}$ be the valuation of $a_{\alpha}(z)$ with respect to $z_{0}$. Hence if $a_{\alpha}(z) \not \equiv 0$, $a_{\alpha}(z)=z_{0}^{\jmath_{a}} b_{\alpha}(z)$ with $b_{\alpha}\left(0, z^{\prime}\right) \not \equiv 0$ and for $a_{\alpha}(z) \equiv 0$ put $j_{\alpha}=+\infty$. Let us proceed to define the characteristic polygon $\Sigma$ of $P(z, \partial)$ with respect to $K=\left\{z_{0}=0\right\}$. Put

$$
e_{\alpha}=j_{\kappa}-\alpha_{0},
$$

where $e_{\alpha}=+\infty$ if $a_{\alpha}(z) \equiv 0$. We denote by $\Pi(a, b)$ the infinite rectangle $\left\{(x, y) \in \mathbb{R}^{2} ; x \leq a, y \geq b\right\}$. The characteristic polygon $\Sigma$ is defined by $\Sigma:=$ the convex hull of $\cup_{\alpha} \Pi\left(|\alpha|, e_{\alpha}\right)$. The boundary of $\Sigma$ consists of a vertical half line $\Sigma(0)$, a horizontal half line $\Sigma(p)$ and $p-1$ segments $\Sigma(i)(1 \leq i \leq p-1)$ with slope $\gamma_{2}, 0=\gamma_{p}<\gamma_{p-1}<\cdots<\gamma_{1}<\gamma_{0}=+\infty$.

Let $\left\{\left(k_{l}, e(i)\right) \in \boldsymbol{R}^{2} ; 0 \leq i \leq p-1\right\}$ be vertices of $\Sigma$, where $0 \leq k_{p-1}<$ $k_{p-2}<\cdots<k_{\imath}<k_{\imath-1}<\cdots<k_{0}=m$. So the endpoints of $\Sigma(i)(1 \leq i \leq p-1)$ are $\left(k_{\imath-1}, e(i-1)\right)$ and $\left(k_{\imath}, e(i)\right)$.

Definition 1.1. The slope $\gamma_{\imath}$ of $\Sigma(i)$ is called the $i$-th characteristic index of $P(z, \partial)$ with respect to $K=\left\{z_{0}=0\right\}$.

The definition of the characteristic indices in this paper is slightly different from that in [4], where the characteristic indices were denoted by $\sigma_{\imath}$, and $\gamma_{\imath}=$ $\sigma_{\imath}-1(0 \leq i \leq p)$ holds.

Now we notice the vertices of the polygon $\Sigma$. So put subsets $\Delta(i)$ of multi-indices and quantities $l_{\imath}(0 \leq i \leq p-1)$ as follows:

$$
\left\{\begin{aligned}
\Delta(i) & :=\left\{\alpha \in \mathbb{N}^{d+1} ;|\alpha|=k_{\iota}, j_{\alpha}-\alpha_{0}=e(i)\right\}, \\
l_{\imath} & :=\max \left\{\left|\alpha^{\prime}\right|: \alpha \in \Delta(i)\right\} .
\end{aligned}\right.
$$

Define a subset $\Delta_{0}(i)$ of $\Delta(i)$

$$
\Delta_{0}(i)=\left\{\alpha \in \Delta(i) ;\left|\alpha^{\prime}\right|=l_{\imath}\right\}
$$




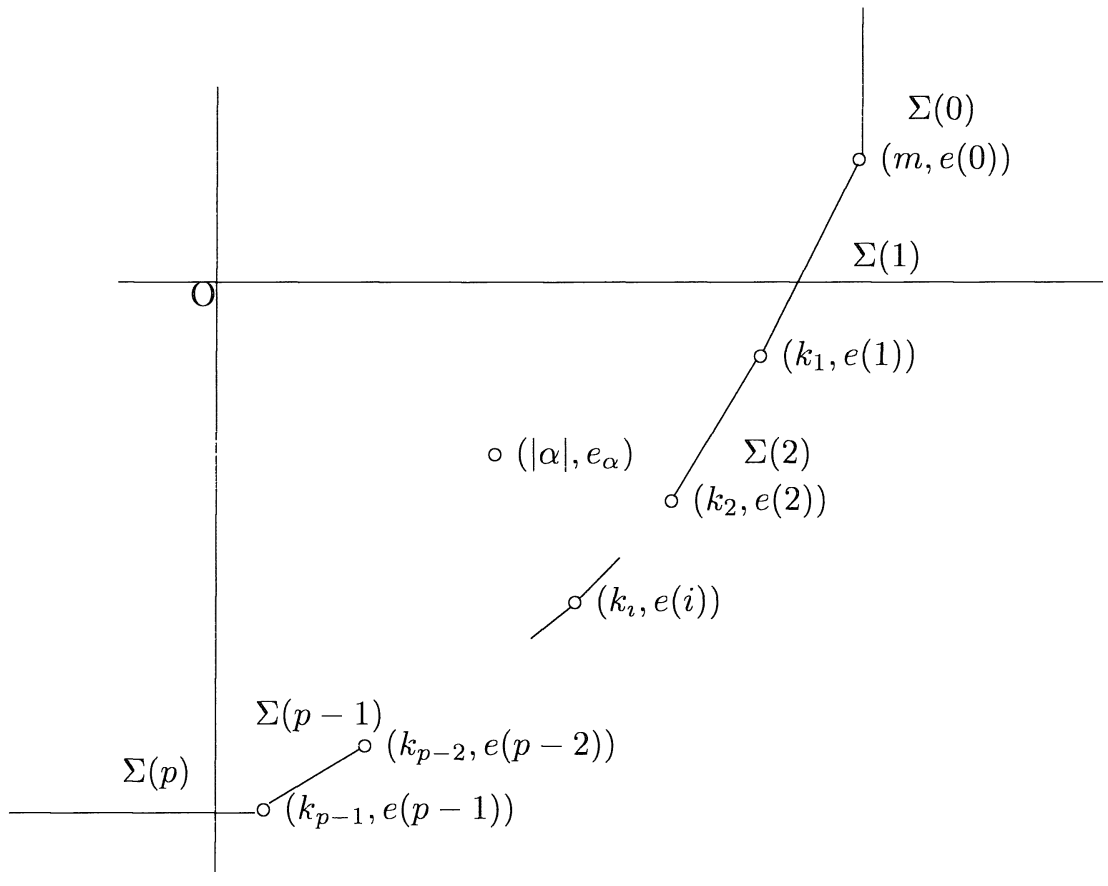

Figure 1. Characteristic polygon

If $\alpha \in \Delta_{0}(i), j_{\alpha}=e(i)-k_{\imath}+l_{\imath}$. So $j_{\alpha}$ does not depend on $\alpha$ for $\alpha \in \Delta_{0}(i)$. Hence we can define a polynomial $\chi_{P, i}\left(z^{\prime}, \xi^{\prime}\right)$ in $\xi^{\prime}$ by

$$
\chi_{P, \imath}\left(z^{\prime}, \xi^{\prime}\right)=\sum_{\alpha \in \Delta_{0}(\imath)} b_{\alpha}\left(0, z^{\prime}\right) \xi^{\alpha^{\prime}},
$$

which is homogeneous in $\xi^{\prime}$ with degree $l_{\imath}$ and plays an important role in the present paper.

Next let us define spaces of holomorphic functions in some regions. Let $\Omega=$ $\Omega_{0} \times \Omega^{\prime}$ be a polydisk with $\Omega_{0}=\left\{z_{0} \in \mathbb{C}^{\prime} ;\left|z_{0}\right|<R\right\}$ and $\Omega^{\prime}=\left\{z^{\prime} \in \mathbb{C}^{d} ;\left|z^{\prime}\right|<\right.$ $R\}$ for some positive constant $R$. Put $\Omega_{0}(\theta)=\left\{z_{0} \in \Omega_{0}-\{0\} ;\left|\arg z_{0}\right|<\theta\right\}$ and $\Omega(\theta)=\Omega_{0}(\theta) \times \Omega^{\prime}$. $\mathcal{O}(\Omega)\left(\mathcal{O}\left(\Omega^{\prime}\right), \mathcal{O}(\Omega(\theta))\right)$ is the set of all holomorphic functions on $\Omega$ (resp. $\Omega^{\prime}, \Omega(\theta)$ ). $\mathcal{O}(\Omega(\theta)$ ) contains multi-valued functions, if $\theta>\pi$.

We introduce the subspaces $\mathcal{O}_{(\kappa)}(\Omega(\theta))$ and $A s y_{\{\kappa\}}(\Omega(\theta))$ of $\mathcal{O}(\Omega(\theta))$.

Definition 1.2. $\mathcal{O}_{(\kappa)}(\Omega(\theta))(0<\kappa \leq+\infty)$ is the set of all $u(z) \in$ 
$\mathcal{O}(\Omega(\theta))$ such that for any $\varepsilon>0$ and any $\theta^{\prime}$ with $0<\theta^{\prime}<\theta$

$$
|u(z)| \leq C \exp \left(\varepsilon\left|z_{0}\right|^{-\kappa}\right) \quad \text { for } z \in \Omega\left(\theta^{\prime}\right)
$$

holds for a constant $C=C\left(\varepsilon, \theta^{\prime}\right)$. We put $\mathcal{O}_{(+\infty)}(\Omega(\theta))=\mathcal{O}(\Omega(\theta))$ for $\kappa=+\infty$.

Definition 1.3. $A s y_{\{\kappa\}}(\Omega(\theta))(0<\kappa \leq+\infty)$ is the set of all $u(z) \in$ $\mathcal{O}(\Omega(\theta))$ such that for any $\theta^{\prime}$ with $0<\theta^{\prime}<\theta$ and any $N \in \mathbb{N}$

$$
\left|u(z)-\sum_{n=0}^{N-1} u_{n}\left(z^{\prime}\right) z_{0}^{n}\right| \leq A B^{N}\left|z_{0}\right|^{N} \Gamma\left(\frac{N}{\kappa}+1\right) \quad z \in \Omega\left(\theta^{\prime}\right),
$$

where $u_{n}\left(z^{\prime}\right) \in \mathcal{O}\left(\Omega^{\prime}\right)(n \in \mathbb{N})$, holds for constants $A=A\left(\theta^{\prime}\right)$ and $B=B\left(\theta^{\prime}\right)$.

We say that $u(z) \in A s y_{\{\kappa\}}(\Omega(\theta))$ has an asymptotic expansion with Gevrey exponent (or index) $\kappa \cdot u(z) \in A s y_{\{+\infty\}}(\Omega(\theta))$ means that $u(z)$ is holomorphic at $z=0$.

We give a condition on $P(z, \partial)$ considered in the present paper. For fixed $i(0 \leq i \leq p-1)$,

$\left(C_{\imath}\right) \quad j_{\alpha}=0$ for all $\alpha \in \Delta_{0}(i)$.

The main result is the following.

Theorem 1.4. Suppose that $P(z, \partial)$ satisfies $\left(C_{\imath}\right)$ and $\chi_{P, \imath}\left(0, \hat{\xi}^{\prime}\right) \neq 0$, $\hat{\xi}^{\prime}=(1,0, \cdots, 0)$. Let $u(z) \in \mathcal{O}_{\left(\gamma_{2}\right)}(\Omega(\theta))$ be a solution of

$$
P(z, \partial) u(z)=f(z) \in A s y_{\left\{\gamma_{2}\right\}}(\Omega(\theta))
$$

such that

$$
\partial_{1}^{h} u\left(z_{0}, 0, z^{\prime \prime}\right) \in A s y_{\left\{\gamma_{2}\right\}}\left(\Omega(\theta) \cap\left\{z_{1}=0\right\}\right) \quad \text { for } \quad 0 \leq h \leq l_{\imath}-1 .
$$

Then there is a polydisk $W$ centered at $z=0$ such that $u(z) \in A s y_{\left\{\gamma_{2}\right\}}(W(\theta))$.

We considered in [6] the case $i=p-1$ and $l_{p-1}=0$ and obtained the same result for this case. If $\left(C_{\imath}\right)$ does not hold, $u(z)$ does not have asymptotic expansion but the behaviors of solutions become less regular. We studied in [7] the behaviors of solutions under the condition that $i=p-1$ and $l_{p-1}=0$ but that $\left(C_{p-1}\right)$ does not always hold.

We give an example. Let us consider

$$
P(z, \partial)=\partial_{1}^{5}+\partial_{1}^{3} \partial_{0}+\partial_{0}^{2}, \quad z=\left(z_{0}, z_{1}\right) \in \mathbb{C}^{2} .
$$


We have

$$
\left\{\begin{array}{l}
\gamma_{0}=+\infty, \quad \gamma_{1}=1, \quad \gamma_{2}=1 / 2, \quad \gamma_{3}=0, \\
\chi_{P .0}\left(z^{\prime}, \xi_{1}\right)=\xi_{1}^{5}, \quad \chi_{P, 1}\left(z^{\prime}, \xi_{1}\right)=\xi_{1}^{3}, \quad \chi_{P, 2}\left(z^{\prime}, \xi_{1}\right)=1 .
\end{array}\right.
$$

Obviously $P(z, \partial)$ satisfies $\left(C_{\imath}\right)$ and $\chi_{P, \imath}\left(z^{\prime}, 1\right) \neq 0$ for $i=0,1,2$. Consequently it follows from Theorem 1.4 that there is a polydisk $W$ centered at $z=0$ such that

$$
\begin{aligned}
i=0: & u(z) \in \mathcal{O}_{(+\infty)}(\Omega(\theta)), \partial_{1}^{h} u\left(z_{0}, 0\right) \in A s y_{\{+\infty\}}\left(\Omega_{0}(\theta)\right)(0 \leq h \leq 4) \quad \text { and } \\
& f(z) \in A s y_{\{+\infty\}}(\Omega(\theta)) \Rightarrow u(z) \in A s y_{\{+\infty\}}(W(\theta)), \\
i=1: & u(z) \in \mathcal{O}_{(1)}(\Omega(\theta)), \partial_{1}^{h} u\left(z_{0}, 0\right) \in A s y_{\{1\}}\left(\Omega_{0}(\theta)\right)(0 \leq h \leq 2) \quad \text { and } \\
& f(z) \in \operatorname{Asy}_{\{1\}}(\Omega(\theta)) \Rightarrow u(z) \in \operatorname{Asy}_{\{1\}}(W(\theta)), \\
i=2: & u(z) \in \mathcal{O}_{(1 / 2)}(\Omega(\theta)), f(z) \in \operatorname{Asy}_{\{1 / 2\}}(\Omega(\theta)) \Rightarrow u(z) \in A s y_{\{1 / 2\}}(W(\theta)) .
\end{aligned}
$$

\section{§2. Estimate of Derivatives}

The purpose of this section is to obtain estimates of the derivatives $\partial_{0}^{N} u\left(z_{0}, z^{\prime}\right)$. By using the obtained estimates, we show Theorem1.4 in the following sections. So first let us study majorant functions. For formal power series of one variable $t, A(t)=\sum_{n=0}^{\infty} A_{n} t^{n}$ and $B(t)=\sum_{n=0}^{\infty} B_{n} t^{n}, A(t) \ll B(t)$ means $\left|A_{n}\right| \leq B_{n}$ for all $n \in \mathbb{N}$. Put

$$
\left\{\begin{array}{l}
\psi^{(k)}(t)=k ! /(r-t)^{k+1} \quad \text { for } \quad k \geq 0 \\
\psi^{(k)}(t)=\int_{0}^{t} \psi^{(k+1)}(\tau) d \tau \quad \text { for } \quad k<0
\end{array}\right.
$$

which satisfy $\frac{d \psi^{(h)}}{d t}(t)=\psi^{(k+1)}(t)$ and if $0<r \leq 1, \psi^{(k)}(t) \ll \psi^{(k+1)}(t)$. By modifying $\psi^{(k)}(t)$, define other majorant functions $\Psi_{k}^{(s)}(t)(k \in \mathbb{Z}, s \in \mathbb{N})$

$$
\Psi_{k}^{(s)}(t)=\left(\frac{d}{d t}\right)^{s}\left\{\frac{R^{\prime}}{R^{\prime}-t} \psi^{(k)}(t)\right\} \quad \text { where } 0<r<R^{\prime}<1 .
$$

We have

Lemma 2.1. (1) The following inequalities hold:

$$
\left\{\begin{array}{l}
\left(R^{\prime}-t\right) \Psi_{k}^{(s)}(t) \gg 0, \quad \Psi_{k}^{(s)}(t) \gg \Psi_{k+1}^{(s-1)}(t), \quad \Psi_{k}^{(s)}(t) \gg \Psi_{k}^{(s-1)}(t), \\
\frac{1}{R-R^{\prime}} \Psi_{k}^{(s)}(t) \gg(R-t)^{-1} \Psi_{k}^{(s)}(t) \quad \text { for } \quad R^{\prime}<R
\end{array}\right.
$$


(2) If $k \geq 0$,

$$
\psi^{(s+k)}(t) \ll \Psi_{k}^{(s)}(t) \ll \frac{R^{\prime}}{R^{\prime}-r} \psi^{(s+k)}(t)
$$

(3) If $k<0$ and $R^{\prime}>2 r$,

$$
\psi^{\left(s+k^{\prime}\right)}(t) \ll \Psi_{k^{\prime}}^{(s)}(t) \ll \frac{2^{\left|k^{\prime}\right|} R^{\prime}}{R^{\prime}-2 r} \psi^{\left(s+k^{\cdot}\right)}(t)
$$

(4) Let $|t| \leq r / 2$. Then

$$
\begin{aligned}
& \left|\psi^{(k)}(t)\right| \leq \frac{2^{k+1} k !}{r^{k+1}} \quad \text { for } \quad k \geq 0 \\
& \left|\psi^{(k)}(t)\right| \leq \frac{2|t||k|}{r|k| !} \quad \text { for } \quad k \leq 0 .
\end{aligned}
$$

(5) Let $|t| \leq r / 2, R^{\prime}>2 r, s \geq 0$ and $k \geq 0$. Then there exist constants $C_{0}$ and $C_{1}$ such that

$$
\left|\Psi_{-h}^{(s)}(t)\right| \leq \frac{C_{0} C_{1}^{k+s} s !}{k !}
$$

The proofs are not difficult and we may refer it to [3] (see also [1] and [8]). However for the convenience of the readers we give them.

Proof. (1) From the definition of $\Psi_{k}^{(0)}(t),\left(R^{\prime}-t\right) \Psi_{k}^{(0)}(t) \gg 0$. So $\left(R^{\prime}-\right.$ $t) \Psi_{k}^{(1)}(t) \gg \Psi_{k}^{(0)}(t) \gg 0$. By induction on $s$ we have $\left(R^{\prime}-t\right) \Psi_{k}^{(s)}(t) \gg 0$. It holds that

$$
\Psi_{k}^{(s)}(t)=R^{\prime}(d / d t)^{s-1}\left(\left(R^{\prime}-t\right)^{-1} \psi^{(k+1)}(t)+\left(R^{\prime}-t\right)^{-2} \psi^{(k)}(t)\right)
$$

So we have the second inequality and the third one. The fourth inequality follows from $(R-t)^{-1}\left(R^{\prime}-t\right) \Psi_{k}^{(s)}(t)=\left(1-\left(R-R^{\prime}\right)(R-t)^{-1}\right) \Psi_{k}^{(s)}(t) \gg 0$.

(2) It is obvious that $\psi^{(s+k)}(t) \ll \Psi_{k}^{(s)}(t)$ for all $k \in \mathbb{Z}$. From (1) we have $\left(R^{\prime}-t\right)^{-1} \psi^{(k)}(t) \ll\left(R^{\prime}-r\right)^{-1} \psi^{(k)}(t)$. Hence the desired inequality follows.

(3) If $k<0$, then $R^{\prime}\left(R^{\prime}-t\right)^{-1} \psi^{(k)}(t)=\sum_{n=0}^{+\infty} C_{n} t^{n+|k|} /\left(r^{n+1}(n+1) \ldots\right.$ $(n+|k|))$, where $C_{n}=\sum_{\imath=0}^{n} \frac{(n+1) \cdots(n+|k|)}{(n-i+1) \cdots(n-i+|k|)}\left(\frac{r}{R^{\prime}}\right)^{\imath}$. Since $C_{0}=1$ 
and for $0<i \leq n$

$$
\begin{aligned}
& \frac{(n+1) \cdots(n+|k|)}{(n-i+1) \cdots(n-i+|k|)}=\frac{(n+|k|-i+1) \cdots(n+|k|)}{(n+1-i) \cdots n} \\
& =\left(1+\frac{|k|}{n+1-i}\right)\left(1+\frac{|k|}{n+2-i}\right) \cdots\left(1+\frac{|k|}{n}\right) \\
& \leq\left(1+\frac{|k|}{1}\right)\left(1+\frac{|k|}{2}\right) \cdots\left(1+\frac{|k|}{i}\right)=\left(\begin{array}{c}
i+|k| \\
i
\end{array}\right) \leq 2^{2+|k|},
\end{aligned}
$$

we have $C_{n} \leq 2^{|k|} R^{\prime} /\left(R^{\prime}-2 r\right)$. Hence $\Psi_{k}(t) \ll 2^{|k|} R^{\prime} /\left(R^{\prime}-2 r\right) \psi^{(k)}(t)$, from which the inequality in (3) follows.

(4) Let $|t| \leq r / 2$. It is easy to show $\left|\psi^{(k)}(t)\right| \leq 2^{k+1} k ! / r^{k+1}$ for $k \geq 0$. Let $k<0$. Then $\left|\psi^{(k)}(t)\right| \leq \sum_{l=0}^{+\infty} \frac{|t|^{l+|k|}}{r^{l+1}(l+1) \cdots(l+|k|)} \leq \frac{|t|^{|k|}}{r|k| !} \sum_{l=0}^{+\infty} \frac{|t|^{l}}{r^{l}} \leq \frac{2|t|^{|k|}}{r|k| !}$.

(5) It follows from (3) and (4) that $\left|\Psi_{-k}^{(s)}(t)\right| \leq 2^{k} R^{\prime}\left|\psi^{(s-k)}(t)\right| /\left(R^{\prime}-2 r\right)$. We have for $s \geq k\left|\psi^{(s-k)}(t)\right| \leq 2^{s-k+1}(s-k) ! / r^{s-k+1} \leq 2^{s-k+1} s ! /\left(r^{s-k+1} k !\right)$ and for $k \geq s\left|\psi^{(s-k)}(t)\right| \leq 2|t|^{k-s} /(r(k-s) !) \leq 2^{k+1}|t|^{k-s} s ! /(r k !)$. Hence there exist constants $C_{0}$ and $C_{1}$ such that $\left|\Psi_{-k}^{(s)}(t)\right| \leq C_{0} C_{1}^{k+s} s ! / k !$.

In order to estimate holomorphic functions in a domain that is sectorial with respect to $z_{0}$, let us introduce a series of majorant functions $\left\{\Psi_{k}^{(s)}(a ; z) ; k \in\right.$ $\mathbb{Z}, s \in \mathbb{N}\}$,

$$
\Psi_{k}^{(s)}(a ; z)=\Psi_{k}^{(s)}\left(\frac{z_{0}-a}{c a}+\rho z_{1}+z_{2}+\cdots+z_{d}\right),
$$

where $0<2 r<R^{\prime}<1, \rho \geq 1,0<a<R / 2$ and $0<c<1$. For a domain $\Omega=\left\{z \in \mathbb{C}^{d+1} ;|z|<R\right\}\left(R^{\prime}<R \leq 1\right)$, put

$$
\Omega(a, c)=\left\{z=\left(z_{0}, z^{\prime}\right) ;\left|z_{0}-a\right| \leq c a R,\left|z^{\prime}\right|<R\right\} .
$$

If $\left|z_{0}-a\right| \leq c a R$, then $\left|z_{0}\right| \leq a+c a R<2 a<R$ and $\Omega(a, c)$ is a subset of $\Omega$. For formal power series $A(z)=\sum_{\alpha} A_{\alpha}\left(z_{0}-a\right)^{\alpha_{0}}\left(z^{\prime}\right)^{\alpha^{\prime}}$ and $B(z)=\sum_{\alpha} B_{\alpha}\left(z_{0}-\right.$ $a)^{\alpha_{0}}\left(z^{\prime}\right)^{\alpha^{\prime}}$ centered at $(a, 0, \cdots, 0), A(z) \underset{\left(a, 0^{\prime}\right)}{\ll} B(z)$ means $\left|A_{\alpha}\right| \leq B_{\alpha}$ for all $\alpha \in \mathbb{N}^{d+1}$. Define an integral operator $\partial_{1}^{-1}$ for $v(z) \in \mathcal{O}(\Omega(\theta))$ as follows:

$$
\partial_{1}^{-1} v(z)=\int_{0}^{z_{1}} v\left(z_{0}, \tau, z^{\prime \prime}\right) d \tau
$$

and $\partial_{1}^{-l} v(z)=\left(\partial_{1}^{-1}\right)^{l} v(z)$ for $l \in \mathbb{N}$.

Lemma 2.2. Let $v(z) \in \mathcal{O}(\Omega(\theta)), 0<\theta^{\prime}<\min \{\theta, \pi / 2\}$ and $c=\sin \theta^{\prime}$. Suppose that $|v(z)| \leq K_{\theta^{\prime}, a}$ on $\Omega(a, c)$. Then $v(z) \underset{\left(a, 0^{\prime}\right)}{\ll} K_{\theta^{\prime}, a}(R-t)^{-1} \underset{\left(a, 0^{\prime}\right)}{\ll}$ $K_{\theta^{\prime}, a} \Psi_{0}^{(0)}(a ; z)$, where $t=\frac{z_{0}-a}{c a}+\rho z_{1}+z_{2}+\cdots+z_{d}$. 
Proof. We have by Cauchy's integral formula

$$
\partial_{0}^{\alpha_{0}} v\left(z_{0}, z^{\prime}\right)=\frac{\alpha_{0} !}{2 \pi i} \oint_{|\zeta-a|=a R \sin \theta^{\prime}} \frac{v\left(\zeta, z^{\prime}\right)}{\left(\zeta-z_{0}\right)^{\alpha_{0}+1}} d \zeta .
$$

Hence $\left|\partial_{0}^{\alpha_{0}} v\left(a, z^{\prime}\right)\right| \leq K_{\theta^{\prime}, a} \alpha_{0} ! /(c a R)^{\alpha_{0}}$ and $\left|\partial^{\alpha} v\left(a, 0^{\prime}\right)\right| \leq K_{\theta^{\prime}, a} \alpha ! /\left((c a)^{\alpha_{0}} R^{|\alpha|}\right)$. Since $0<2 r<R^{\prime}<R \leq 1$,

$$
\begin{aligned}
v(z) & \underset{\left(a, 0^{\prime}\right)}{\ll} K_{\theta^{\prime}, a} /\left(1-\frac{z_{0}-a}{c a R}-\frac{\rho z_{1}+z_{2}+\cdots+z_{d}}{R}\right) \\
& \underset{\left(a, 0^{\prime}\right)}{\ll} K_{\theta^{\prime}, a} /\left(r-\left(\frac{z_{0}-a}{c a}+\rho z_{1}+z_{2}+\cdots+z_{d}\right)\right) \underset{\left(a, 0^{\prime}\right)}{\ll} K_{\theta^{\prime}, a} \Psi_{0}^{(0)}(a ; z) .
\end{aligned}
$$

Lemma 2.3. Let $a(z)=z_{0}^{\jmath} b(z)(j \in \mathbb{N})$ be a holomorphic function on $\Omega$ such that $|b(z)| \leq B$. Then $a(z) \underset{\left(a, 0^{\prime}\right)}{\ll} 2^{\jmath} B R a^{\jmath}(R-t)^{-1}$ and

$$
a(z) \partial^{\alpha} \Psi_{k}^{(s)}(a ; z) \underset{\left(a, 0^{\prime}\right)}{\ll} \frac{2^{\jmath} B R \rho^{\alpha_{1}} a^{\jmath-\alpha_{0}}}{\left(R-R^{\prime}\right) c^{\alpha_{0}}} \Psi_{k}^{(s+|\alpha|)}(a ; z),
$$

where $t=\frac{z_{0}-a}{c a}+\rho z_{1}+z_{2}+\cdots+z_{d}$.

Proof. We have $|a(z)| \leq((c R+1) a)^{\jmath} B \leq 2^{\jmath} B a^{\jmath}$ on $\Omega(a, c)$ and by Lemma $2.2 a(z) \underset{\left(a, 0^{\prime}\right)}{\ll} 2^{\jmath} B R a^{\jmath}(R-t)^{-1}$. Since $\partial^{\alpha} \Psi_{k}^{(s)}(a ; z)=\rho^{\alpha_{1}}(c a)^{-\alpha_{0}} \Psi_{k}^{(s+|\alpha|)}(a ; z)$, we have (2.11) by the last inequality in (2.3) in Lemma 2.1 .

Lemma 2.4. Let $w(z)$ be a solution of

$$
\left\{\begin{aligned}
\partial_{1}^{l} w(z) & =f(z), \\
\partial_{1}^{h} w\left(z_{0}, 0, z^{\prime \prime}\right) & =0 \quad \text { for } 0 \leq h \leq l-1 .
\end{aligned}\right.
$$

If $f(z) \underset{\left(a, 0^{\prime}\right)}{\ll} \Psi_{k}^{(\jmath+l)}(a ; z)$, then $w(z) \underset{\left(a, 0^{\prime}\right)}{\ll} \rho^{-l} \Psi_{k}^{(\jmath)}(a ; z)$.

Proof. The estimate of $w(z)$ follows from $\partial_{1}^{l} \rho^{-l} \Psi_{k}^{(\jmath)}(a ; z)=\Psi_{k}^{(\jmath+l)}(a ; z)$.

Lemma 2.5. Let $u(z) \in A s y_{\{\gamma\}}(\Omega(\theta))(0<\gamma \leq+\infty)$. Then $\partial_{0}^{h} u(z) \in$ $\operatorname{Asy}_{\{\gamma\}}(\Omega(\theta))$ for $h \in \mathbb{N}$ and for any $0<\theta^{\prime}<\theta$ there are constants $M=M\left(\theta^{\prime}\right)$ and $C=C\left(\theta^{\prime}\right)$ such that $\left|\partial_{0}^{h} u(z)\right| \leq M C^{h} \Gamma\left(\frac{h}{\delta}+1\right), \delta=\gamma /(\gamma+1)$, in $\Omega\left(\theta^{\prime}\right)$. 
Proof. Suppose $u(z)$ has the asymptotic expansion (1.7) with $\kappa=\gamma$. Then it follows from Cauchy's integral formula that $\partial_{0}^{h} u(z) \in \operatorname{Asy}_{\{\gamma\}}(\Omega(\theta))$ and for any $0<\theta^{\prime}<\theta$ there are constants $A_{\imath}=A_{\imath}\left(\theta^{\prime}\right)(i=1,2,3,4)$ such that $\left|\partial_{0}^{h} u(z)-\sum_{n=0}^{N-1}(n+h) \cdots(n+1) u_{n+h}\left(z^{\prime}\right) z_{0}^{n}\right| \leq A_{1}^{h+1} A_{2}^{N}\left|z_{0}\right|^{N} h ! \Gamma\left(\frac{N+h}{\gamma}+1\right) \leq$ $A_{3}^{h+1} \Gamma\left(\frac{h}{\delta}+1\right) A_{4}^{N}\left|z_{0}\right|^{N} \Gamma\left(\frac{N}{\gamma}+1\right)$ and $\left|\partial_{0}^{h} u(z)\right| \leq A_{3}^{h+1} \Gamma\left(\frac{h}{\delta}+1\right)$ in $\Omega\left(\theta^{\prime}\right)$.

Now let us return to

$$
P(z, \partial) u(z)=f(z)
$$

The coefficients of $P(z, \partial)$ are holomorphic in a domain containing $\bar{\Omega}=\{z \in$ $\left.\mathbb{C}^{d+1} ;|z| \leq R\right\}$. Suppose that $P(z, \partial)$ satisfies $\left(C_{\imath}\right)$. Then $j_{\alpha}=0$ for all $\alpha \in$ $\Delta_{0}(i)$ and $e(i)=-k_{\imath}+l_{\imath} \cdot \chi_{P, 2}\left(z^{\prime}, \xi^{\prime}\right)=\sum_{\alpha \in \Delta_{0}(\imath)} b_{\alpha}\left(0, z^{\prime}\right) \xi^{\alpha^{\prime}}$ is a homogeneous polynomial in $\xi^{\prime}$ with degree $l_{\imath}$. Further suppose that $\chi_{P, \imath}\left(0, \hat{\xi}^{\prime}\right) \neq 0, \hat{\xi}^{\prime}=$ $(1,0, \cdots, 0)$. Put $\alpha(i)=\left(-e(i), \alpha^{\prime}(i)\right) \in \mathbb{N} \times \mathbb{N}^{d}, \alpha^{\prime}(i)=\left(l_{\imath}, 0, \cdots, 0\right)$. Then $b_{\alpha(\imath)}(0) \neq 0$. Define $m^{*}(i)=\max _{\left\{\alpha ; e_{\alpha}<+\infty\right\}}\left(e_{\alpha}-e(i)\right)$.

Proposition 2.6. Suppose that $P(z, \partial)$ satisfies $\left(C_{\imath}\right)$ and $\chi_{P, z}\left(z^{\prime}, \hat{\xi}^{\prime}\right) \neq 0$ on $\bar{\Omega}^{\prime}=\left\{z^{\prime} \in \mathbb{C}^{d} ;\left|z^{\prime}\right| \leq R\right\}, \hat{\xi}^{\prime}=(1,0, \cdots, 0)$. Let $u(z) \in \mathcal{O}(\Omega(\theta))$ be a solution of $(\mathrm{Eq})$ with $\partial_{1}^{l} u\left(z_{0}, 0, z^{\prime \prime}\right)=0$ for $0 \leq l \leq l_{\imath}-1$ and $f(z) \in \operatorname{Asy}_{\left\{\gamma_{\imath}\right\}}(\Omega(\theta))$. Let $0<\theta^{\prime}<\min \{\theta, \pi / 2\}$ and $c=\sin \theta^{\prime}$. Put $M(a, c)=\sup \left\{\left|\partial_{0}^{n} u(z)\right| ; z \in\right.$ $\left.\Omega(a, c), 0 \leq n<\max \left\{-e(i), m^{*}(i)\right\}\right\}$.

Then there are constants $A=A\left(\theta^{\prime}\right), B$ and $\rho=\rho\left(\theta^{\prime}\right) \geq 1$ which do not depend on a such that the following estimates hold: if $0 \leq i \leq p-2$,

$\partial_{0}^{n} \partial_{1}^{l_{2}} u(z) \underset{\left(a ; 0^{\prime}\right)}{\ll} M(a, c) A^{n+1}\left(\sum_{h=0}^{+\infty} B^{h}(n+1)^{h}\left(\sum_{h^{\prime}=0}^{+\infty} \frac{\Psi_{-h-\left[h^{\prime} / \gamma_{2}+1\right]}^{\left(\left[n\left(1+1 / \gamma_{2}\right)+m\right)\right.}(a ; z)}{\mid a_{\mid}^{\mid h^{\prime}}}\right)\right)$,

where $1 /+\infty=0$ for $i=0$, and if $i=p-1$,

$$
\partial_{0}^{n} \partial_{1}^{l_{p-1}} u(z) \underset{\left(a, 0^{\prime}\right)}{\ll} M(a, c) A^{n+1}\left(\sum_{h=0}^{+\infty} B^{h}(n+1)^{h} \Psi_{-h}^{\left(\left[n\left(1+1 / \gamma_{2}\right)\right]+m\right)}(a ; z)\right)
$$

for $n \in \mathbb{N}$, where $r, R^{\prime}, R$ are small positive constants with $2 r<R^{\prime}<R<1$.

Proof. The assumption $\chi_{P, \imath}\left(z^{\prime}, \hat{\xi}^{\prime}\right) \neq 0$ means $a_{\alpha(\imath)}\left(0, z^{\prime}\right)=b_{\alpha(\imath)}\left(z^{\prime}\right) \neq 0$. So we may assume $a_{\alpha(\imath)}(z)=1$. Put $v(z):=\partial_{1}^{l_{l}} u(z)$ and consider $P(z, \partial) \partial_{1}^{-l_{\imath}} v(z)$. 
We decompose $P(z, \partial) \partial_{1}^{-l_{\iota}}$ into two parts,

$$
\left\{\begin{aligned}
P(z, \partial) \partial_{1}^{-l_{\iota}} & =Q(z, \partial)+R(z, \partial) \\
Q(z, \partial) & =\sum_{\left\{a ; e_{\alpha}>e(\imath)\right\}} a_{\alpha}(z) \partial^{\alpha} \partial_{1}^{-l_{\imath}} \\
R(z, \partial) & =\sum_{\left\{\alpha ; e_{\alpha} \leq e(\imath)\right\}} a_{\alpha}(z) \partial^{\alpha} \partial_{1}^{-l_{\imath}}
\end{aligned}\right.
$$

We show (2.13) by induction on $n$. We may assume by Lemma 2.2 that (2.13) holds for $0 \leq n \leq N-1$ with $N \geq \max \left\{-e(i), m^{*}(i)\right\}$. By differentiating $a_{\alpha}(z) \partial^{\alpha} \partial_{1}^{-l_{l}} v(z)(N+e(i))$ - times with respect to $z_{0}$, $\partial_{0}^{N+e(\imath)}\left(a_{\alpha}(z) \partial^{\alpha} \partial_{1}^{-l_{\imath}} v(z)\right)=\sum_{r=0}^{N+e(\imath)}\left(\begin{array}{c}N+e(i) \\ r\end{array}\right) \partial_{0}^{r} a_{\alpha}(z) \partial^{\alpha^{\prime}} \partial_{1}^{-l_{\imath}} \partial_{0}^{N+\alpha_{0}+e(\imath)-r} v(z)$

First let us estimate $\partial_{0}^{N+e(\imath)} Q(z, \partial) v(z)$. So let $\alpha$ be a multi-index with $e_{\alpha}>e(i)$. Suppose $r \geq j_{\alpha}$. Then $N+\alpha_{0}+e(i)-r \leq N+e(i)-e_{\alpha} \leq N-1$. Hence by the inductive hypothesis, Lemma 2.3 and Lemma 2.4 there is a constant $C_{0}$ such that

$$
\begin{aligned}
\partial_{0}^{r} a_{\alpha}(z) \partial^{\alpha^{\prime}} \partial_{1}^{-l_{l}} \partial_{0}^{N+\alpha_{0}+e(\imath)-r} v(z) & \underset{\left(a, 0^{\prime}\right)}{\ll} \quad M(a, c) A^{N} \rho^{\alpha_{1}-l_{\imath}} C_{0}^{r+1} r !\left(\sum_{h=0}^{+\infty} B^{h} N^{h}\right. \\
& \left.\times\left(\sum_{h^{\prime}=0}^{+\infty} \frac{\Psi_{-h-\left[h^{\prime} / \gamma_{l+1}\right]}^{\left(\left[\left(N+\alpha_{0}+e(\imath)-r\right)\left(1+1 / \gamma_{\imath}\right)\right]+\left|\alpha^{\prime}\right|-l_{\imath}+m\right)}(a ; z)}{|a|^{h^{\prime}}}\right)\right) .
\end{aligned}
$$

We have, by $\left(e_{\alpha}-e(i)\right) / \gamma_{\imath} \geq|\alpha|-k_{\imath}$,

$$
\begin{aligned}
& {\left[\left(N+\alpha_{0}+e(i)-r\right)\left(1+1 / \gamma_{\imath}\right)\right]+\left|\alpha^{\prime}\right|-l_{\imath} } \\
= & {\left[N\left(1+1 / \gamma_{\imath}\right)+\left(e(i)-e_{\alpha}-r+j_{\alpha}\right)\left(1+1 / \gamma_{\imath}\right)\right]+\left|\alpha^{\prime}\right|-l_{\imath} } \\
= & {\left[N\left(1+1 / \gamma_{\imath}\right)+\left(e(i)-e_{\alpha}-r+j_{\alpha}\right) / \gamma_{\imath}\right]+\left|\alpha^{\prime}\right|+e(i)-e_{\alpha}-r+j_{\alpha}-l_{\imath} } \\
= & {\left[N\left(1+1 / \gamma_{\imath}\right)+\left(e(i)-e_{\alpha}-r+j_{\alpha}\right) / \gamma_{\imath}\right]+|\alpha|-k_{\imath}-r } \\
\leq & {\left[N\left(1+1 / \gamma_{\imath}\right)-\left(r-j_{\alpha}\right) / \gamma_{\imath}\right]-r \leq\left[N\left(1+1 / \gamma_{\imath}\right)\right]-r . }
\end{aligned}
$$


Hence by Lemma 2.1 we have for $r \geq j_{\alpha}$

$$
\begin{aligned}
& \partial_{0}^{r} a_{\alpha}(z) \partial^{\alpha^{\prime}} \partial_{1}^{-l_{\iota}} \partial_{0}^{N+\alpha_{0}+e(\imath)-r} v(z) \\
& \underset{\left(a, 0^{\prime}\right)}{\ll} M(a, c) A^{N} \rho^{\alpha_{1}-l_{\iota}} C_{0}^{r+1} r !\left(\sum_{h=0}^{+\infty} B^{h} N^{h}\left(\sum_{h^{\prime}=0}^{+\infty} \frac{\Psi_{-h-\left[h^{\prime} / \gamma_{2}+1\right]}^{\left(\left[N\left(1+1 / \gamma_{\imath}\right)\right]-r+m\right)}(a ; z)}{|a|^{h^{\prime}}}\right)\right) \\
& \underset{\left(a, 0^{\prime}\right)}{\ll} M(a, c) A^{N} \rho^{\alpha_{1}-l_{\iota}} C_{0}^{r+1} r !\left(\sum_{h=0}^{+\infty} B^{h} N^{h}\left(\sum_{h^{\prime}=0}^{+\infty} \frac{\Psi_{-h-r-\left[h^{\prime} / \gamma_{2}+1\right.}^{\left(\left[N\left(1+1 / \gamma_{2}\right)\right]+m\right)}(a ; z)}{|a|^{h^{\prime}}}\right)\right) .
\end{aligned}
$$

Suppose $0 \leq r<j_{\alpha}$. Then $\partial_{0}^{r} a_{\alpha}(z)=O\left(\left|z_{0}\right|^{\jmath_{a}-r}\right)$. By the assumption $N \geq$ $m^{*}(i)$ and $e_{\alpha}-e(i)>0$, we have $0 \leq N+e(i)-e_{\alpha} \leq N-1$ and $\partial_{0}^{N+\alpha_{0}+e(\imath)-r}=$ $\partial_{0}^{\jmath_{a}-r} \partial_{0}^{N+e(\imath)-e_{a}}$. So by Lemmas 2.3 and 2.4

$$
\begin{gathered}
\partial_{0}^{r} a_{\alpha}(z) \partial^{\alpha^{\prime}} \partial_{1}^{-l_{\imath}} \partial_{0}^{N+\alpha_{0}+e(\imath)-r} v(z) \\
\underset{\left(a, 0^{\prime}\right)}{\ll} M(a, c) A^{N} \rho^{\alpha_{1}-l_{\iota}} C_{0}^{r+1} r ! c^{r-\jmath_{\alpha}}\left(\sum_{h=0}^{+\infty} B^{h} N^{h}\right. \\
\left.\times\left(\sum_{h^{\prime}=0}^{+\infty} \frac{\Psi_{-h-\left[h^{\prime} / \gamma_{2}+1\right.}^{\left.\left(\left[(2)-e_{\alpha}\right)\left(1+1 / \gamma_{\iota}\right)\right]+\left|\alpha^{\prime}\right|+\jmath_{a}-r-l_{\imath}+m\right)}(a ; z)}{|a|^{h^{\prime}}}\right)\right) .
\end{gathered}
$$

We have, by the relation $\left(e_{\alpha}-e(i)\right) / \gamma_{\imath} \geq|\alpha|-k_{\imath}$,

$$
\begin{aligned}
& {\left[\left(N+e(i)-e_{\alpha}\right)\left(1+1 / \gamma_{\imath}\right)\right]+\left|\alpha^{\prime}\right|+j_{\alpha}-r-l_{\imath} } \\
\leq & {\left[N\left(1+1 / \gamma_{\imath}\right)\right]+\left(e(i)-e_{\alpha}\right)+k_{\imath}-\alpha_{0}+j_{\alpha}-r-l_{\imath} } \\
\leq & {\left[N\left(1+1 / \gamma_{\imath}\right)\right]-r }
\end{aligned}
$$

and

$$
\begin{gathered}
\Psi_{-h-\left[h^{\prime} / \gamma_{2}+1\right]}^{\left(\left[\left(N+e(2)-e_{\alpha}\right)\left(1+1 / \gamma_{2}\right)\right]+\left|\alpha^{\prime}\right|+\jmath_{\alpha}-r-l_{\imath}+m\right)}(a ; z) \\
\ll \Psi_{\left.-h-\left[h^{\prime} / \gamma_{2}\right)\right]}^{\left(\left[N\left(1+1 / \gamma_{2}\right)\right]-r+m\right)}(a ; z) \ll \Psi_{-h-r-\left[h^{\prime} / \gamma_{c+1}\right]}^{\left(\left[N\left(1+1 / \gamma_{1}\right)\right]+m\right)}(a ; z) .
\end{gathered}
$$

Thus we have for $0 \leq r<j_{\alpha}$

$$
\begin{aligned}
& \partial_{0}^{r} a_{\alpha}(z) \partial^{\alpha^{\prime}} \partial_{1}^{-l_{\imath}} \partial_{0}^{N+\alpha_{0}+e(\imath)-r} v(z) \underset{\left(a, 0^{\prime}\right)}{\ll} M(a, c) A^{N} \rho^{\alpha_{1}-l_{2}} C_{0}^{r+1} r ! c^{r-\jmath_{\alpha}} \\
& \times\left(\sum_{h=0}^{+\infty} B^{h} N^{h}\left(\sum_{h^{\prime}=0}^{+\infty} \frac{\Psi_{-h-r-\left[h^{\prime} / \gamma_{2}+1\right]}^{\left(\left[N\left(1+1 / \gamma_{\imath}\right]+m\right)\right.}(a ; z)}{|a|^{h^{\prime}}}\right)\right) .
\end{aligned}
$$

Hence, by choosing $B$ such that $B>2 C_{0}$ and Lemma 2.1 , there are constants $A_{0}=A_{0}(\rho, c)$ and $A_{1}=A_{1}(\rho, c)$ such that 


$$
\begin{aligned}
& \partial_{0}^{N+e(\imath)} Q(z, \partial) v(z) \\
& \underset{\left(a, 0^{\prime}\right)}{\ll} M(a, c) A_{0} A^{N}\left(\sum_{r=0}^{N+e(\imath)}\left(\begin{array}{c}
N+e(i) \\
r
\end{array}\right) C_{0}^{r+1} r !\right. \\
& \left.\times\left(\sum_{l=0}^{+\infty} B^{l} N^{l}\left(\sum_{h^{\prime}=0}^{+\infty} \frac{\Psi_{-l-r-\left[h^{\prime} / \gamma_{2+1}\right]}^{\left(\left[N\left(1+1 / \gamma_{2}\right)+m\right)\right.}(a ; z)}{|a|^{h^{\prime}}}\right)\right)\right) \\
& =M(a, c) A_{0} A^{N} C_{0}\left(\sum_{r=0}^{N+e(\imath)} \frac{(N+e(i)) ! C_{0}^{r}}{(N+e(i)-r) !}\right. \\
& \left.\times\left(\sum_{l=0}^{+\infty} B^{l} N^{l}\left(\sum_{h^{\prime}=0}^{+\infty} \frac{\Psi_{-l-r-\left[h^{\prime} / \gamma_{l+1}\right]}^{\left(\left[N\left(1+1 / \gamma_{l}\right)+m\right)\right.}(a ; z)}{|a|^{h^{\prime}}}\right)\right)\right)
\end{aligned}
$$

$\underset{\left(a, 0^{\prime}\right)}{\ll} M(a, c) A_{0} A^{N} C_{0}$

$$
\begin{aligned}
&\left(\sum_{r=0}^{N+e(\imath)}\left(N C_{0}\right)^{r}\left(\sum_{l=0}^{+\infty} B^{l} N^{l}\left(\sum_{h^{\prime}=0}^{+\infty} \frac{\Psi_{-l-r-\left[h^{\prime} / \gamma_{l+1}\right]}^{\left(\left[N\left(1+1 / \gamma_{2}\right)\right]+m\right)}(a ; z)}{|a|^{h^{\prime}}}\right)\right)\right. \\
& \underset{\left(a, 0^{\prime}\right)}{\ll} M(a, c) A_{0} A^{N} C_{0}\left(\sum_{h=0}^{+\infty} N^{h}\left(\sum_{l+r=h} C_{0}^{r} B^{l}\right)\left(\sum_{h^{\prime}=0}^{+\infty} \frac{\Psi_{-h-\left[h^{\prime} / \gamma_{1+1}\right]}^{\left(\left[N\left(1+1 / \gamma_{2}\right]+m\right)\right.}(a ; z)}{|a|^{h^{\prime}}}\right)\right) \\
&=M(a, c) A_{0} A^{N} C_{0}\left(\sum_{h=0}^{+\infty} B^{h} N^{h}\left(\sum_{r=0}^{h}\left(\frac{C_{0}}{B}\right)^{r}\right)\left(\sum_{h^{\prime}=0}^{+\infty} \frac{\Psi_{-h-\left[h^{\prime} / \gamma_{2}+1\right]}^{\left(\left[N\left(1+1 / \gamma_{1}\right)\right]+m\right)}(a ; z)}{|a|^{h^{\prime}}}\right)\right) \\
& \underset{\left(a, 0^{\prime}\right)}{\ll} M(a, c) A_{1} A^{N}\left(\sum_{h=0}^{+\infty} B^{h} N^{h}\left(\sum_{h^{\prime}=0}^{+\infty} \frac{\Psi_{-h-\left[h^{\prime} / \gamma_{1}+1\right]}^{\left(\left[N\left(1+1 / \gamma_{2}\right)\right]+m\right)}(a ; z)}{|a|^{h^{\prime}}}\right) .\right.
\end{aligned}
$$

Next let us consider $\partial_{0}^{N+e(\imath)} R(z, \partial) v(z)$. We divide it into two parts, $\partial_{0}^{N+e(\imath)} R(z, \partial) v(z)=R_{0}^{N}(z, \partial) v(z)+R_{1}^{N}(z, \partial) v(z)$,

$$
\left\{\begin{array}{c}
R_{0}^{N}(z, \partial) v(z) \\
=\sum_{\left\{\alpha ; e_{\alpha} \leq e(\imath)\right\}} \sum_{r=0}^{\min \left\{\jmath_{\alpha}, N+e(\imath)\right\}}\left(\begin{array}{c}
N+e(i) \\
r
\end{array}\right) \partial_{0}^{r} a_{\alpha}(z) \partial^{\alpha^{\prime}} \partial_{1}^{-l,} \partial_{0}^{N+\alpha_{0}+e(\imath)-r} v(z), \\
R_{1}^{N}(z, \partial) v(z) \\
=\sum_{\left\{\alpha ; e_{\alpha} \leq e(\imath)\right\}} \sum_{\jmath_{\alpha}<r \leq N+e(\imath)}\left(\begin{array}{c}
N+e(i) \\
r
\end{array}\right) \partial_{0}^{r} a_{\alpha}(z) \partial^{\alpha^{\prime}} \partial_{1}^{-l_{\iota}} \partial_{0}^{N+\alpha_{0}+e(\imath)-r} v(z),
\end{array}\right.
$$


where if $j_{\alpha}>N+e(i), R_{1}^{N}(z, \partial) v(z)=0$. Let us estimate $R_{1}^{N}(z, \partial) v(z)$. We have

$$
\begin{aligned}
\partial_{0}^{r} a_{\alpha}(z) \partial^{\alpha^{\prime}} \partial_{1}^{-l_{2}} \partial_{0}^{\alpha_{0}+e(\imath)-\jmath_{a}} \partial_{0}^{N-r+\jmath_{\alpha}} v(z) & \underset{\left(a, 0^{\prime}\right)}{\ll} \quad M(a, c) A^{N} C_{0}^{r+1} r ! \rho^{\alpha_{1}-l_{\imath}} \\
& \left(\sum_{h=0}^{+\infty} B^{h} N^{h} \partial_{0}^{\alpha_{0}+e(\imath)-\jmath_{a}}\left(\sum_{h^{\prime}=0}^{+\infty} \frac{\Psi_{-h-\left[h^{\prime} / \gamma_{2}+1\right]}^{\left(\left[\left(N-r+\jmath_{\alpha}\right)\left(1+1 / \gamma_{2}\right)\right]+\left|\alpha^{\prime}\right|-l_{\imath}+m\right)}(a ; z)}{|a|^{h^{\prime}}}\right)\right) \\
\underset{\left(a, 0^{\prime}\right)}{\ll} \quad M(a, c) A^{N} C_{0}^{r+1} r ! \rho^{\alpha_{1}-l_{\imath}} c^{-\left(e(\imath)-e_{\alpha}\right)} & \\
& \left(\sum_{h=0}^{+\infty} B^{h} N^{h}\left(\sum_{h^{\prime}=0}^{+\infty} \frac{\Psi_{-h-\left[h^{\prime} / \gamma_{2}+1\right]}^{\left(\left[\left(N-r+\jmath_{\alpha}\right)\left(1+1 / \gamma_{2}\right)\right]+|\alpha|-k_{\imath}-\jmath_{\alpha}+m\right)}(a ; z)}{|a|^{h^{\prime}+e(\imath)-e_{\alpha}}}\right) .\right.
\end{aligned}
$$

Since

$$
\begin{aligned}
& {\left[\left(N-r+j_{\alpha}\right)\left(1+1 / \gamma_{\imath}\right)\right]+|\alpha|-k_{\imath}-j_{\alpha} } \\
\leq & {\left[N\left(1+1 / \gamma_{\imath}\right)\right]-r+|\alpha|-k_{\imath} } \\
\leq & {\left[N\left(1+1 / \gamma_{\imath}\right)\right]-r-\left(e(i)-e_{\alpha}\right) / \gamma_{\imath+1}, }
\end{aligned}
$$

we have

$$
\Psi_{-h-\left[h^{\prime} / \gamma_{2}+1\right]}^{\left(\left[\left(N-r+\jmath_{\alpha}\right)\left(1+1 / \gamma_{2}\right)\right]+|\alpha|-k_{2}-\jmath_{\alpha}+m\right)}(a ; z) \underset{\left(a, 0^{\prime}\right)}{\ll} \Psi_{-r-h-\left[\left(h^{\prime}+e(\imath)-e_{\alpha}\right) / \gamma_{\imath+1}\right]}^{\left(\left[N\left(1+1 / \gamma_{2}\right)\right]+m\right)}(a ; z) .
$$

Hence we have, by choosing $B$ with $B>2 C_{0}$,

$$
\begin{aligned}
& R_{1}^{N}(z, \partial) v(z) \\
& \underset{\left(a, 0^{\prime}\right)}{\ll} M(a, c) A_{0} A^{N}\left(\sum_{\left\{\alpha ; e_{\mathrm{a}} \leq e(\imath)\right\}} \sum_{r=\jmath_{\mathrm{a}}+1}^{N+e(\iota)} \frac{(N+e(i)) !}{(N+e(i)-r) !} C_{0}^{r+1}\right. \\
& \left(\sum_{h=0}^{+\infty} B^{h} N^{h}\left(\sum_{h^{\prime}=0}^{+\infty} \frac{\Psi_{-r-h-\left[\left(h^{\prime}+e(\imath)-e_{\alpha}\right) / \gamma_{\iota+1}\right]}^{\left(\left[N\left(1+1 / \gamma^{\prime}\right)\right]+m\right)}(a ; z)}{|a|^{h^{\prime}+e(\imath)-e_{\alpha}}}\right)\right) \\
& \underset{\left(a, 0^{\prime}\right)}{\ll} \quad M(a, c) A_{0} A^{N} C_{0}\left(\sum_{\left\{\alpha ; e_{\alpha} \leq e(\imath)\right\}} \sum_{r=\jmath_{\alpha}+1}^{N+e(\imath)} N^{r} C_{0}^{r}\right. \\
& \left(\sum_{h=0}^{+\infty} B^{h} N^{h}\left(\sum_{h^{\prime}=0}^{+\infty} \frac{\Psi_{-r-h-\left[\left(h^{\prime}+e(\imath)-e_{\alpha}\right) / \gamma_{\imath+1}\right]}^{\left(\left[N\left(1+1 / \gamma_{2}\right)\right]+m\right)}(a ; z)}{|a|^{h^{\prime}+e(\imath)-e_{\alpha}}}\right)\right)
\end{aligned}
$$




$$
\begin{aligned}
& \underset{\left(a, 0^{\prime}\right)}{\ll} M(a, c) A_{0} A^{N} C_{0}\left(\sum_{\left\{\alpha ; e_{a} \leq e(\imath)\right\}}\left(\sum_{r>0}\left(\frac{C_{0}}{B}\right)^{r}\right)\right. \\
&\left(\sum_{h^{\prime \prime}=0}^{+\infty} \sum_{h^{\prime}=0}^{+\infty} B^{h^{\prime \prime}} N^{h^{\prime \prime}} \frac{\Psi_{-h^{\prime \prime}-\left[\left(h^{\prime}+e(2)-e_{\alpha}\right) / \gamma_{1+1}\right]}^{\left(\left[N\left(1+1 / \gamma_{\imath}\right]+m\right)\right.}}{\left.\left.|a|^{h^{\prime}+e(\imath)-e_{a}}\right)\right)}\right. \\
& \underset{\left(a, 0^{\prime}\right)}{\ll} \quad M(a, c) A_{1} A^{N}\left(\sum_{h=0}^{+\infty} B^{h} N^{h}\left(\sum_{h^{\prime}=0}^{+\infty} \frac{\Psi_{-h-\left[h^{\prime} / \gamma_{\imath}+1\right]}^{\left(\left[N\left(1+1 / \gamma_{2}\right)\right]+m\right)}(a ; z)}{|a|^{h^{\prime}}}\right)\right)
\end{aligned}
$$

for some constants $A_{0}=A_{0}(\rho, c)$ and $A_{1}=A_{1}(\rho, c)$. As for the estimate of the derivatives of $f(z)$ it follows from Lemma 2.5 that for any $0<\theta^{\prime}<\theta$

$$
\left|\partial_{0}^{h} f(z)\right| \leq M C^{h} \Gamma\left(h\left(1+\frac{1}{\gamma_{\imath}}\right)+1\right)
$$

holds for $z \in \Omega\left(\theta^{\prime}\right)$. Therefore we have

$$
\left\{\begin{array}{l}
R_{0}^{N}(z, \partial) v(z)=F_{N}(z) \\
F_{N}(z)=-\left(\partial_{0}^{N+e(\imath)} Q(z, \partial)+R_{1}^{N}(z, \partial)\right) v(z)+\partial_{0}^{N+e(\imath)} f(z),
\end{array}\right.
$$

where there is a constant $A_{1}=A_{1}(\rho, c)$ such that

$$
\begin{aligned}
F_{N}(z) & \underset{\left(a, 0^{\prime}\right)}{\ll} M(a, c) A_{1} A^{N} \\
& \left(\sum_{h=0}^{+\infty} B^{h} N^{h}\left(\sum_{h^{\prime}=0}^{+\infty} \frac{\Psi_{-h-\left[h^{\prime} / \gamma_{l}+1\right]}^{\left(\left[N\left(1+1 / \gamma_{2}\right)\right]+m\right)}(a ; z)}{|a|^{h^{\prime}}}\right)\right) .
\end{aligned}
$$

Finally let us obtain the estimate of $\partial_{0}^{N} v(z)$ by using the equation (2.20). We have, by the assumption $a_{\alpha(\imath)}(z)=1$,

$$
\begin{aligned}
& R_{0}^{N}(z, \partial) v(z) \\
& \quad=\sum_{\left\{\alpha ; e_{n} \leq e(\imath)\right\}} \sum_{r=0}^{\min \left\{J_{\alpha}, N+e(\imath)\right\}}\left(\begin{array}{c}
N+e(i) \\
r
\end{array}\right) \partial_{0}^{r} a_{\alpha}(z) \partial^{\alpha^{\prime}} \partial_{1}^{-l_{\imath}} \partial_{0}^{N+\alpha_{0}+e(\imath)-r} v(z) \\
& \quad=\left(I+K_{N}(z, \partial)\right) \partial_{0}^{N} v(z),
\end{aligned}
$$

where $I$ is the identity operator and

$$
K_{N}(z, \partial)=\sum^{\prime}\left(\begin{array}{c}
N+e(i) \\
r
\end{array}\right) \partial_{0}^{r} a_{\alpha}(z) \partial^{\alpha^{\prime}} \partial_{1}^{-l_{\imath}} \partial_{0}^{\alpha_{0}+e(\imath)-r} .
$$


Here $\sum^{\prime}$ means the sum with respect to $(\alpha, r) \neq(\alpha(i), 0)$ with $e_{\alpha} \leq e(i)$ and $0 \leq r \leq \min \left\{j_{\alpha}, N+e(i)\right\}$. Let $\alpha$ be a multi-index appearing in $\sum^{\prime}$. Then $e(i)-e_{\alpha}=e(i)-j_{\alpha}+\alpha_{0} \geq 0, r \leq j_{\alpha}$ and $|\alpha| \leq k_{\imath}$. Hence $\alpha_{0}+e(i)-r \geq 0$. Let us show $l_{\imath}>\alpha_{1}$. It holds that

$$
l_{\imath}=k_{\imath}+e(i) \geq k_{\imath}+e_{\alpha}=k_{\imath}+j_{\alpha}-\alpha_{0}=k_{\imath}+j_{\alpha}-|\alpha|+\left|\alpha^{\prime}\right| \geq\left|\alpha^{\prime}\right| .
$$

So if $e(i)>e_{\alpha}$ or $k_{\imath}>|\alpha|$ or $j_{\alpha}>0$, we have $l_{\imath}>\left|\alpha^{\prime}\right| \geq \alpha_{1}$. Let $\alpha$ be a multi-index with $e(i)=e_{\alpha},|\alpha|=k_{\imath}$ and $j_{\alpha}=0$. Then $\alpha=\left(-e(i), \alpha^{\prime}\right) \in \Delta_{0}(i)$ with $\left|\alpha^{\prime}\right|=l_{\imath}$ and $\alpha \neq \alpha(i)$. Hence $\left|\alpha^{\prime}\right|>\alpha_{1}$. Thus we have $l_{\imath}>\alpha_{1}$ for $\alpha$ appearing in the sum $\sum^{\prime}$.

It follows from (2.20) and (2.22) that

$$
\partial_{0}^{N} v(z)=\sum_{s=0}^{+\infty}\left(-K_{N}(z, \partial)\right)^{s} F_{N}(z) .
$$

Let us estimate $K_{N}(z, \partial) F_{N}(z)$. We have from (2.21)

$$
\begin{aligned}
& \sum^{\prime}\left(\begin{array}{c}
N+e(i) \\
r
\end{array}\right) \partial_{0}^{r} a_{a}(z) \partial^{\alpha^{\prime}} \partial_{1}^{-l_{\imath}} \partial_{0}^{\alpha_{0}+e(\imath)-r} F_{N}(z) \\
& \underset{\left(a, 0^{\prime}\right)}{\ll} M(a, c) A_{1} A^{N} \sum^{\prime}\left(\rho^{\alpha_{1}-l_{\imath}} C_{0}^{r+1} c^{-e(\imath)-\alpha_{0}+r}\right. \\
& \left.\left(\sum_{h=0}^{+\infty} B^{h} N^{h+r}\left(\sum_{h^{\prime}=0}^{+\infty} \frac{\Psi_{-h-\left[h^{\prime} / \gamma_{\iota}+1\right]}^{\left(\left[N\left(1+1 / \gamma_{2}\right)\right]+i \alpha \mid-k_{\imath}-r+m\right.}(a ; z)}{|a|^{h^{\prime}+e(\imath)-e_{\alpha}}}\right)\right)\right) \\
& \underset{\left(a, 0^{\prime}\right)}{\ll} M(a, c) A_{1} A^{N} \rho^{-1} \sum^{\prime}\left(C_{0}^{r+1} c^{-e(\imath)-\alpha_{0}+r}\right. \\
& \left(\sum_{h=0}^{+\infty} B^{h} N^{h+r}\left(\sum_{h^{\prime}=0}^{+\infty} \frac{\Psi_{-h-r-\left[\left(e(2)-e_{\alpha}+h^{\prime}\right) / \gamma_{2+1}\right]}^{\left(\left[N\left(1+1 / \gamma_{2}\right)\right]+|\alpha| k_{2}+m\right.}(a ; z)}{|a|^{h^{\prime}+e(\imath)-e_{\alpha}}}\right)\right),
\end{aligned}
$$

where $A_{1}=A_{1}(\rho, c)$ in (2.21). Hence, by choosing $B>2 C_{0}$, there is a constant $C=C(c)$ which is independent of $\rho$ such that

$$
\begin{aligned}
& K_{N}(z, \partial) F_{N}(z) \\
& \underset{\left(a, 0^{\prime}\right)}{\ll} M(a, c) \rho^{-1} A_{1}(\rho, c) A^{N}\left(\sum^{\prime} c^{-e(\imath)-\alpha_{0}+r} C_{0}^{r+1}\right. \\
&\left.\quad\left(\sum_{h=0}^{+\infty} B^{h} N^{h+r}\left(\sum_{h^{\prime}=0}^{+\infty} \frac{\Psi_{-h-r-\left[\left(e(l)-e_{a}+h^{\prime}\right) / \gamma_{\imath+1}\right]}^{\left(\left[N\left(1+1 / \gamma_{2}\right)\right]+m\right)}}{|a|^{h^{\prime}+e(\imath)-e_{a}}}\right)\right)\right)
\end{aligned}
$$




$$
\underset{\left(a, 0^{\prime}\right)}{\ll} M(a, c) C \rho^{-1} A_{1} A^{N}\left(\sum_{h=0}^{+\infty} B^{h} N^{h}\left(\sum_{h^{\prime}=0}^{+\infty} \frac{\Psi_{-h-\left[h^{\prime} / \gamma_{2}+1\right]}^{\left(\left[N\left(1+1 / \gamma_{2}\right)\right]+m\right)}(a ; z)}{!} a_{\left.\right|^{\mid h^{\prime}}}^{\left(h^{\prime}\right.}\right)\right) .
$$

By repeating this process, we obtain

$$
\begin{aligned}
& \left(K_{N}(z, \partial)\right)^{s} F_{N}(z) \underset{\left(a, 0^{\prime}\right)}{\ll} M(a, c)\left(C \rho^{-1}\right)^{s} A_{1} A^{N} \\
& \left(\sum_{h=0}^{+\infty} B^{h} N^{h}\left(\sum_{h^{\prime}=0}^{+\infty} \frac{\Psi_{-h-\left[h^{\prime} / \gamma_{2}+1\right]}^{\left(\left[N\left(1+1 / \gamma_{2}\right)\right]+m\right)}(a ; z)}{|a|^{h^{\prime}}}\right)\right) .
\end{aligned}
$$

By choosing $\rho, A$ so that $C \rho^{-1}<1 / 2$ and $A>2 A_{1}(\rho, c)$, we have

$$
\begin{aligned}
\partial_{0}^{N} v(z) & =\sum_{s=0}^{\infty}\left(K_{N}(z, \partial)\right)^{s} F_{N}(z) \\
& \underset{\left(a, 0^{\prime}\right)}{\ll} M(a, c) A^{N+1}\left(\sum_{h=0}^{+\infty} B^{h}(N+1)^{h}\left(\sum_{h^{\prime}=0}^{+\infty} \frac{\Psi_{-h-\left[h^{\prime} / \gamma_{2}+1\right]}^{\left(\left[N\left(1+1 / \gamma_{2}\right)\right]+m\right)}(a ; z)}{|a|^{h^{\prime}}}\right)\right)
\end{aligned}
$$

which means (2.13) for $n=N$.

If $i=p-1$, then $e_{\alpha} \geq e(p-1)$ for all $\alpha$. So

$$
R_{0}^{N}(z, \partial) v(z)=\sum_{\left\{\alpha ; e_{\alpha}=e(p-1)\right\}} \sum_{0 \leq r \leq J_{a}}\left(\begin{array}{c}
N+e(i) \\
r
\end{array}\right) \partial_{0}^{r} a_{\alpha}(z) \partial^{\alpha^{\prime}} \partial_{1}^{-l_{\imath}} \partial_{0}^{N+\alpha_{0}+e(\imath)-r} v(z)
$$

and we have the estimate (2.14) by modifying a little the above arguments and noting that the sum is taken for only $\alpha$ with $e_{\alpha}=e(p-1)$ in $R_{0}(z, \partial)$ and $R_{1}(z, \partial)$.

Corollary 2.7. Suppose the assumptions in Proposition 2.6 hold. Further assume $u(z) \in \mathcal{O}_{\left(\gamma_{1}\right)}(\Omega(\theta))$. Then if $i=0, u(z)$ is holomorphic at $z=0$ and if $i>0$, there is a polydisk $W$ centered at $z=0$ such that for any $\varepsilon>0$ and any $\theta^{\prime}$ with $0<\theta^{\prime}<\theta$ there are constants $C=C\left(\theta^{\prime}\right)$ and $M_{\varepsilon}=M_{\varepsilon}\left(\theta^{\prime}\right)$ such that

$$
\left|\partial_{0}^{n} u\left(z_{0}, z^{\prime}\right)\right| \leq M_{\varepsilon} C^{n} \exp \left(\varepsilon\left|z_{0}\right|^{-\gamma_{\imath}}\right) \Gamma\left(n\left(\frac{\gamma_{\imath}+1}{\gamma_{\imath}}\right)+1\right) \quad \text { for } \quad z \in W\left(\theta^{\prime}\right)
$$

and $n=0,1, \cdots$. 
Proof. Let $|t| \leq r / 2$. Then it follows from Lemma 2.1 that there are constants $C_{0}$ and $C_{1}$ such that if $0 \leq i \leq p-2$,

$$
\left|\Psi_{-h-\left[h^{\prime} / \gamma_{\imath}+1\right]}^{\left(\left[n\left(1+1 / \gamma_{\iota}\right)\right]+m\right)}(t)\right| \leq C_{0} C_{1}^{n+h+h^{\prime}} \frac{\Gamma\left(n\left(1+1 / \gamma_{\imath}\right)+1\right)}{\Gamma(h+1) \Gamma\left(\left[h^{\prime} / \gamma_{\imath+1}\right]+1\right)}
$$

and if $i=p-1$,

$$
\left|\Psi_{-h}^{\left(\left[n\left(1+1 / \gamma_{\imath}\right)\right]+m\right)}(t)\right| \leq C_{0} C_{1}^{n+h} \frac{\Gamma\left(n\left(1+1 / \gamma_{\imath}\right)+1\right)}{\Gamma(h+1)} .
$$

Let $W_{0}=\left\{z_{0} \in \mathbb{C} ;\left|z_{0}\right|<R / 2\right\}, W^{\prime}=\left\{z^{\prime} \in \mathbb{C}^{d} ;\left|z_{1}\right|<r / 2 \rho(d+1),\left|z_{\imath}\right|<\right.$ $r / 2(d+1), 2 \leq i \leq d\}$ and $W=W_{0} \times W^{\prime}$. Let $0<a<R / 2,0<\theta_{0}<$ $\min \{\theta, \pi / 2\}, c=\sin \theta_{0}$ and $\left|\left(z_{0}-a\right) /(c a)\right|<r / 2(d+1)$. Then $\left|\frac{z_{0}-a}{c a}\right|+$ $\left|\rho z_{1}\right|+\left|z_{2}\right|+\cdots+\left|z_{d}\right|<r / 2$ for $z^{\prime} \in W^{\prime}$. Hence it follows from Proposition 2.6 that for $z \in\left\{z_{0} ;\left|\frac{z_{0}-a}{c a}\right|<r / 2(d+1)\right\} \times W^{\prime}$

$$
\begin{aligned}
& \left|\partial_{0}^{n} \partial_{1}^{l_{1}} u(z)\right| \leq M(a, c) C_{0}\left(A C_{1}\right)^{n+1} \Gamma\left(n\left(1+\frac{1}{\gamma_{\imath}}\right)+1\right) \\
& \left(\sum_{h=0}^{+\infty} \frac{\left(B C_{1}\right)^{h}(n+1)^{h}}{\Gamma(h+1)}\right)\left(\sum_{h^{\prime}=0}^{+\infty} \frac{C_{1}^{h^{\prime}}}{|a|^{h^{\prime}} \Gamma\left(\left[h^{\prime} / \gamma_{2+1}\right]+1\right)}\right) \\
& \leq M(a, c) C_{0}\left(A C_{1}\right)^{n+1} C_{2}^{n+1} \exp \left(C^{*}|a|^{-\gamma_{2+1}}\right) \Gamma\left(n\left(1+\frac{1}{\gamma_{2}}\right)+1\right)
\end{aligned}
$$

for some constants $C_{2}>0$ and $C^{*} \geq 0$, where if $i=p-1, C^{*}=0$. So if $i=0$, then $\gamma_{0}=+\infty$ and

$$
\left|\partial_{0}^{n} \partial_{1}^{l_{2}} u(z)\right| \leq M(a, c) \exp \left(C^{*}|a|^{-\gamma_{1+1}}\right) C_{0} C_{3}{ }^{n+1} n !,
$$

which means the holomorphy of $u(z)$ at $z=0$, by considering the conditions on the traces $\partial_{1}^{l} u(z)\left(0 \leq l<l_{\imath}\right)$ on $z_{1}=0$. Suppose that $i>0$ and $u(z) \in$ $\mathcal{O}_{\left(\gamma_{l}\right)}(\Omega(\theta))$. Then for any $\varepsilon>0$ and $\theta^{\prime \prime}$ with $\theta_{0}<\theta^{\prime \prime}<\theta$, there is a constant $K_{\varepsilon}=K_{\varepsilon}\left(\theta^{\prime \prime}\right)$ such that $M(a, c) \leq K_{\varepsilon} \exp \left(\varepsilon|a|^{-\gamma_{2}} / 2\right)$. Hence

$$
\left|\partial_{0}^{n} \partial_{1}^{l_{2}} u(z)\right| \leq K_{\varepsilon} \exp \left(\varepsilon|a|^{-\gamma_{\imath}} / 2+C^{*}|a|^{-\gamma_{\imath+1}}\right) C_{0} C_{3}{ }^{n+1} \Gamma\left(n\left(1+\frac{1}{\gamma_{\imath}}\right)+1\right) .
$$

Therefore we have from the conditions of the traces of $\partial_{l}^{l} u(z)\left(0 \leq l<l_{\imath}\right)$ on $z_{1}=0$

$$
\left|\partial_{0}^{n} u(z)\right| \leq M_{\varepsilon} \exp \left(\varepsilon|a|^{-\gamma_{\imath}}\right) C^{n} \Gamma\left(n\left(1+\frac{1}{\gamma_{\imath}}\right)+1\right)
$$


and, by putting $z_{0}=a$,

$$
\left|\partial_{0}^{n} u\left(a, z^{\prime}\right)\right| \leq M_{\varepsilon} \exp \left(\varepsilon|a|^{-\gamma_{\imath}}\right) C^{n} \Gamma\left(n\left(1+\frac{1}{\gamma_{\imath}}\right)+1\right) .
$$

In the above we assume $a>0$. If $\left(a, z^{\prime}\right) \in W\left(\theta^{\prime}\right)$, by the transformation $u_{\phi}\left(z_{0}, z^{\prime}\right)=u\left(z_{0} e^{\imath \phi}, z^{\prime}\right) \quad(\phi=\arg a)$, we can reduce this case to the preceding. Hence we have (2.26).

\section{$\S 3 . \quad$ Asymptotic Expansion}

In order to complete the proof of Theorem 1.4 we require the following theorem, which was given in [6],

Theorem 3.1. Let $U$ be a polydisk in $\mathbb{C}^{d+1}$ with center $z=0$ and $0<$ $\gamma \leq+\infty$. Suppose that $u(z) \in \mathcal{O}(U(\theta))$ satisfies the following estimate: for any $\varepsilon>0$ and $0<\theta^{\prime}<\theta$ there exist constants $M_{\varepsilon}=M\left(\varepsilon, \theta^{\prime}\right)$ and $C=C\left(\theta^{\prime}\right)$ such that

$$
\left|\left(\frac{\partial}{\partial z_{0}}\right)^{n} u(z)\right| \leq M_{\varepsilon} \exp \left(\varepsilon\left|z_{0}\right|^{-\gamma}\right) C^{n} \Gamma\left(n\left(\frac{\gamma+1}{\gamma}\right)+1\right) \quad \text { for } \quad z \in U\left(\theta^{\prime}\right)
$$

for all $n \in \mathbb{N}$. Then $u(z) \in A s y_{\{\gamma\}}(W(\theta))$ for some polydisk $W$ with center $z=0$.

Theorem 1.4 immediately follows from Corollary 2.7 and Theorem 3.1. In [6] we showed Theorem 3.1 for rational $\gamma$, where we reduced the proof to the analysis of some simple partial differential equation of Fuchsian type. In this paper we show it for real $\gamma$ and give a different proof which Prof. Honda (Hokkaido Univ.) suggested to the author. Here we use a differential operator with infinite order and its inverse operator, which are similar to those in [2].

Only the variable $z_{0}$ is essential in Theorem 3.1. So in the following discussion we treat functions in one variable and regard other variables as parameters. First we give

Lemma 3.2. Let $g(t)$ be a continuous function on $[0, T](T>0)$ and $\kappa$ be a positive constant. Suppose that there exist positive constants $M$ and $c$ such that for any $m \in \mathbb{N}$

$$
|g(t)| \leq M c^{m} t^{m} \Gamma\left(\frac{m}{\kappa}+1\right) \text { on }[0, T] .
$$

Then $|g(t)| \leq C_{0} M(c t)^{-\kappa / 2} e^{-(c t)^{-\kappa}}$ holds for a constant $C_{0}$ that is independent of $M$ and $c$. 
Proof. First we assume $c=1$. Let $m \in \mathbb{N}$ with $\kappa / m \leq T^{\kappa}$. Suppose that $\kappa /(m+1) \leq t^{\kappa} \leq \kappa / m$. Then, by (3.2) and Stiring's formula $\Gamma(m / \kappa+1) \sim$ $\left(\frac{m}{\kappa}\right)^{m / \kappa} \sqrt{2 \pi \frac{m}{\kappa}} e^{-m / \kappa}$, there is a constant $C_{0}$ such that

$$
|g(t)| \leq M\left(\frac{\kappa}{m}\right)^{\frac{m}{\kappa}} \Gamma\left(\frac{m}{\kappa}+1\right) \leq C_{0} M \sqrt{\frac{m}{\kappa}} e^{-(m+1) / \kappa} \leq C_{0} M \frac{e^{-t^{-\kappa}}}{t^{\kappa / 2}} .
$$

This means $|g(t)| \leq C_{0} M t^{-\kappa / 2} \exp \left(-t^{-\kappa}\right)$ for $t \in[\kappa /(m+1), \kappa / m]$ and for all $m \in \mathbb{N}$ with $\kappa / m \leq T^{\kappa}$. So the assertion holds for $c=1$. By considering $g(t / c)$, we have the estimate of $g(t)$ for general $c>0$.

Now put

$$
A(\lambda)=\prod_{n=1}^{+\infty}\left(1+\frac{\lambda}{n^{1+\frac{1}{\sim}}}\right) .
$$

Lemma 3.3. (i) $A(\lambda)$ is an entire function with estimate

$$
\mid A(\lambda) \leq C_{0} \exp \left(c_{0}|\lambda|^{\gamma /(\gamma+1)}\right) .
$$

(ii) $1 / A(\lambda)$ is holomorphic in $\mathbb{C}-(-\infty,-1]$ and there are positive constants $C_{1}$ and $c_{1}$ such that

$$
|A(\lambda)|^{-1} \leq C_{1} \exp \left(-c_{1}|\lambda|^{\gamma /(\gamma+1)}\right) \quad \text { for } \quad \Re \lambda \geq 0
$$

and for $0<\theta^{\prime}<\pi$ there are positive constants $C_{2}=C\left(\theta^{\prime}\right)$ and $c_{2}=c_{2}\left(\theta^{\prime}\right)$ such that

$$
|A(\lambda)|^{-1} \leq C_{2} \exp \left(c_{2}|\lambda|^{\gamma /(\gamma+1)}\right) \quad \text { for } \quad|\arg \lambda| \leq \theta^{\prime}
$$

Proof. (i) Let $q^{1+1 / \gamma} \leq|\lambda| \leq(q+1)^{1+1 / \gamma}(q \in \mathbb{N})$. Then

$$
\begin{aligned}
\prod_{n=1}^{q}(1+ & \left.\frac{|\lambda|}{n^{1+1 / \gamma}}\right) \prod_{n=q+1}^{+\infty}\left(1+\frac{|\lambda|}{n^{1+1 / \gamma}}\right) \\
\leq & \exp \left(\sum_{n=q+1}^{+\infty} \frac{|\lambda|}{n^{1+1 / \gamma}}\right) \prod_{n=1}^{q}\left(1+\frac{|\lambda|}{n^{1+1 / \gamma}}\right) \\
\leq & \exp \left(\sum_{n=q+1}^{+\infty}\left(\frac{q+1}{n}\right)^{1+1 / \gamma}\right) \prod_{n=1}^{q}\left(1+\frac{|\lambda|}{n^{1+1 / \gamma}}\right) \\
\leq & \exp \left(C^{\prime}(q+1)\right) \prod_{n=1}^{q}\left(1+\frac{\mid \lambda^{\mid}}{n^{1+1 / \gamma}}\right) \\
& \left.\quad \sum_{n=q+1}^{+\infty} \frac{1}{n^{1+1 / \gamma}} \leq C^{\prime}(q+1)^{-1 / \gamma}\right)
\end{aligned}
$$




$$
\leq \exp \left(C^{\prime}(q+1)\right) \frac{2^{q}|\lambda|^{q}}{(q !)^{1+1 / \gamma}} \leq A B^{q} \frac{|\lambda|^{q}}{(q !)^{1+1 / \gamma}} \leq C_{0} \exp \left(c_{0}|\lambda|^{\gamma /(\gamma+1)}\right) .
$$

(ii) Let $\Re \lambda \geq 0$. Then $\left|1+\frac{\lambda}{n^{1+1 / \gamma}}\right| \geq 1$. Hence

$$
\prod_{n=1}^{+\infty}\left|1+\frac{\lambda}{n^{1+1 / \gamma}}\right| \geq \sup _{l} \prod_{n=1}^{l}\left|1+\frac{\lambda}{n^{1+1 / \gamma}}\right| \geq \sup _{l} \frac{|\lambda|^{l}}{(l !)^{1+1 / \gamma}} .
$$

If $q^{1+1 / \gamma} \leq|\lambda| \leq(q+1)^{1+1 / \gamma}(q \in \mathbb{N})$, then we have by the Stiring's formula $q ! \sim(2 \pi q)^{1 / 2} q^{q} e^{-q}$

$$
\frac{|\lambda|^{q}}{(q !)^{1+1 / \gamma}} \geq \frac{q^{q(1+1 / \gamma)}}{(q !)^{1+1 / \gamma}} \geq C^{\prime} \exp \left(c^{\prime} q\right) \geq C_{1}^{-1} \exp \left(c_{1}|\lambda|^{\gamma /(\gamma+1)}\right)
$$

for some positive constants $C^{\prime}, C_{1}, c$ and $c^{\prime}$, from which (3.5) follows.

Finally let us show the estimate (3.6). Let $n(x)=\left[x^{\gamma /(\gamma+1)}\right]$ for $x \geq 0$. Then, by integration by parts, we have

$\log A(\lambda)=\int_{0}^{+\infty} \log \left(1+\frac{\lambda}{x}\right) d n(x)=\lambda \int_{0}^{+\infty} \frac{n(x) d x}{(\lambda+x) x}=\lambda \int_{0}^{+\infty} \frac{N(x)}{(x+\lambda)^{2}} d x$ where $N(x)=\int_{(0, x]} \frac{n(t)}{t} d t$. Since $N(x) \leq C x^{\gamma /(\gamma+1)}$, there is a constant $c_{2}=c_{2}\left(\theta^{\prime}\right)$ such that for $\lambda$ with $|\arg \lambda|<\theta^{\prime}<\pi$

$$
\begin{aligned}
& \left.|\log A(\lambda) ! \leq C| \lambda\left|\int_{0}^{+\infty} \frac{x^{\gamma /(\gamma+1)}}{|x+\lambda|^{2}} d x \leq C\right| \lambda\right|^{2+\gamma /(\gamma+1)} \int_{0}^{+\infty} \frac{t^{\gamma /(\gamma+1)}}{|i \lambda| t+\lambda_{i}^{2}} d t \\
& =C|\lambda|^{\gamma /(\gamma+1)} \int_{0}^{+\infty} \frac{t^{\gamma /(\gamma+1)}}{\mid t+e^{\left.2 \arg \lambda\right|^{2}}} d t \leq c_{2}|\lambda|^{\gamma /(\gamma+1)}
\end{aligned}
$$

which means (3.6).

It follows from (3.4) that $A(\lambda)$ is an entire function with exponential order $\gamma /(\gamma+1)$. So by the theory of entire functions we have

$$
A(\lambda)=\sum_{n=0}^{+\infty} a_{n} \lambda^{n}, \quad\left|a_{n}\right| \leq \frac{A B^{n}}{\Gamma\left(n\left(\frac{\gamma+1}{\gamma}\right)+1\right)}
$$

for some constants $A$ and $B$. Hence we can define a differential operator $A(d / d t)$ with infinite order that operates on holomorphic functions: $A(d / d t) u(t)=$ $\sum_{n=0}^{+\infty} a_{n}(d / d t)^{n} u(t)$. 
Define

$$
K(t)=\frac{1}{2 \pi i} \int_{0}^{\infty e^{2 \varphi}} \frac{\exp (\lambda t)}{A(\lambda)} d \lambda,
$$

where $|\arg t+\varphi-\pi|<\pi / 2$ and $|\varphi|<\pi$.

Lemma 3.4. (i) $K(t)$ is holomorphic in $\mathbb{C}_{(-\pi / 2,5 \pi / 2)}=\{t \neq 0 ;-\pi / 2<$ $\arg t<5 \pi / 2\}$ and

$$
A(d / d t) K(t)=\frac{-1}{2 \pi i t} .
$$

(ii) Let $0 \leq \arg t \leq 2 \pi$. Then there are constants $A$ and $D$ such that

$$
\left|\left(\frac{d}{d t}\right)^{n} K(t)\right| \leq A D^{n} \Gamma\left((n+1)\left(\frac{\gamma+1}{\gamma}\right)\right) .
$$

(iii) There exist positive constants $A, D_{0}$ and $c$ such that

$$
\left|\left(\frac{d}{d t}\right)^{n}\left(K(t)-K\left(t e^{2 \pi \imath}\right)\right)\right| \leq A D_{0}^{n} \exp \left(-c t^{-\gamma}\right) \Gamma\left((n+1)\left(\frac{\gamma+1}{\gamma}\right)\right) \text { for } t \geq 0 .
$$

Proof. (i) It follows from (3.6) that $K(t)$ is holomorphic in $\mathbb{C}_{(-\pi / 2,5 \pi / 2)}$. It is obvious that $K(t)$ satisfies (3.9).

(ii) For $t$ with $0 \leq \arg t \leq 2 \pi$ we can choose $|\varphi| \leq \pi / 2$ such that $\mid \arg t+\varphi-$ $\pi \mid \leq \pi / 2$. Hence, by Lemma 3.3-(ii), we have

$$
\begin{aligned}
& \left|\left(\frac{d}{d t}\right)^{n} K(t)\right|=\frac{1}{2 \pi}\left|\int_{0}^{\infty e^{i \varphi}} \frac{\lambda^{n} \exp (\lambda t)}{A(\lambda)} d \lambda\right| \\
& \quad \leq A_{1} \int_{0}^{+\infty} r^{n} \exp \left(-c_{1} r^{\gamma /(\gamma+1)}\right) d r \leq A D^{n} \Gamma\left((n+1)\left(\frac{\gamma+1}{\gamma}\right)\right) .
\end{aligned}
$$

(iii) Let $t>0$. Then we have

$$
\begin{aligned}
\left(\frac{d}{d t}\right)^{l}\left(K(t)-K\left(t e^{2 \pi \imath}\right)\right) & =\frac{1}{2 \pi i}\left(\int_{0}^{\infty e^{2 \pi / 2}}-\int_{0}^{\infty e^{-\imath \pi / 2}}\right) \frac{\lambda^{l} \exp (\lambda t)}{A(\lambda)} d \lambda \\
& =\frac{1}{2 \pi i} \int_{\infty e^{-\imath \pi / 2}}^{\infty e^{2 \pi / 2}} \frac{\lambda^{l} \exp (\lambda t)}{A(\lambda)} d \lambda
\end{aligned}
$$


So

$$
\lim _{t \rightarrow+0}\left(\frac{d}{d t}\right)^{l}\left(K(t)-K\left(t e^{2 \pi \imath}\right)\right)=\frac{1}{2 \pi i} \int_{\infty e^{-\imath \pi / 2}}^{\infty e^{2 \pi / 2}} \lambda^{l} A(\lambda)^{-1} d \lambda=0
$$

and

$$
\begin{aligned}
\left|\left(\frac{d}{d t}\right)^{l}\left(K(t)-K\left(t e^{2 \pi \imath}\right)\right)\right| & \leq A_{1} \int_{-\infty}^{+\infty}|r|^{l} \exp \left(-c_{1}|r|^{\gamma /(\gamma+1)}\right) d r \\
& \leq A_{2} D^{l} \Gamma\left((l+1)\left(\frac{\gamma+1}{\gamma}\right)\right) .
\end{aligned}
$$

Hence it follows from Taylor's expansion of $\left(\frac{d}{d t}\right)^{n}\left(K(t)-K\left(t e^{2 \pi \imath}\right)\right.$ that there are constants $A_{3}$ and $D_{0}$ such that

$$
\begin{aligned}
\mid\left(\frac{d}{d t}\right)^{n} & \left(K(t)-K\left(t e^{2 \pi \imath}\right) \mid\right. \\
& \leq \frac{|t|^{m}}{m !}\left|\left(\frac{d}{d t}\right)^{n+m}\left(K(\theta t)-K\left(\theta t e^{2 \pi \imath}\right)\right)\right| \quad(0<\theta<1) \\
& \leq A_{2} D^{m+n} \Gamma\left((m+n+1)\left(\frac{\gamma+1}{\gamma}\right)\right) t^{m} / m ! \\
& \leq A_{3} D_{0}^{m+n} \Gamma\left((n+1)\left(\frac{\gamma+1}{\gamma}\right)\right) \Gamma\left(\frac{m}{\gamma}+1\right) t^{m}
\end{aligned}
$$

for any $m \in \mathbb{N}$. So by Lemma 3.2 there exist positive constants $A$ and $c$ such that $\left|\left(\frac{d}{d t}\right)^{n}\left(K(t)-K\left(t e^{2 \pi \imath}\right)\right)\right| \leq A D_{0}^{n} \Gamma\left((n+1)\left(\frac{\gamma+1}{\gamma}\right)\right) \exp \left(-c t^{-\gamma}\right)$ for $t \geq 0$.

Now let $U_{0}=\left\{w_{0} ;\left|w_{0}\right|<R\right\}, V_{0}=\left\{z_{0} ;\left|z_{0}\right|<R / 2\right\}$ and $0<\theta<\pi / 2$. Let $0<\theta_{0}<\theta, u\left(w_{0}\right) \in \mathcal{O}\left(U_{0}(\theta)\right)$ and $z_{0} \in V_{0}\left(\theta_{0}\right)$. Define

$$
(K u)\left(z_{0}\right)=\frac{1}{2 \pi i} \int_{\mathcal{C}} K\left(w_{0}-z_{0}\right) u\left(w_{0}\right) d w_{0},
$$

where $\mathcal{C}$ is a closed path in $U_{0}(\theta)$ in $w_{0}$-space, which starts at a fixed point $w_{0}=$ $a, 3 R / 4 \leq a<R$, encloses once the point $w_{0}=z_{0}, z_{0}=x_{0}+i y_{0}$, anticlockwise and ends at point $w_{0}=a$. We may take $\mathcal{C}$ so that $-\pi / 2<\arg \left(w_{0}-z_{0}\right)<5 \pi / 2$ for $w_{0} \in \mathcal{C}$. We have

Proposition 3.5. (i) $(K u)\left(z_{0}\right) \in \mathcal{O}\left(V_{0}\left(\theta_{0}\right)\right)$ and

$$
\left\{\begin{array}{l}
A\left(-\partial_{z_{0}}\right)(K u)\left(z_{0}\right)=-u\left(z_{0}\right) \\
A\left(-\partial_{z_{0}}\right)(K u)\left(z_{0}\right)=\frac{1}{2 \pi i} \int_{\mathcal{C}} K\left(w_{0}-z_{0}\right) A\left(-\partial_{w_{0}}\right) u\left(w_{0}\right) d w_{0}+\tilde{u}\left(z_{0}\right)
\end{array}\right.
$$


where $\tilde{u}\left(z_{0}\right)$ is holomorphically extensible to a neighborhood of the origin.

(ii) If $u\left(z_{0}\right) \in \mathcal{O}_{(\gamma)}\left(U_{0}(\theta)\right)$, then $(K u)\left(z_{0}\right) \in A s y_{\{\gamma\}}\left(V_{0}\left(\theta_{0}\right)\right)$.

Proof. (i) It is obvious that $(K u)\left(z_{0}\right) \in \mathcal{O}\left(V_{0}\left(\theta_{0}\right)\right)$. By Lemma 3.4-(i),

$$
A\left(-\partial_{z_{0}}\right)\left(K u\left(z_{0}\right)\right)=\frac{1}{2 \pi i} \int_{\mathcal{C}} \frac{-u_{0}\left(w_{0}\right) d w_{0}}{w_{0}-z_{0}}=-u\left(z_{0}\right) .
$$

On the other hand we have

$$
\begin{aligned}
\left(-\partial_{z_{0}}\right)^{n}(K u)\left(z_{0}\right) & =\frac{1}{2 \pi i} \int_{\mathcal{C}}\left(\left(\partial_{w_{0}}\right)^{n} K\left(w_{0}-z_{0}\right)\right) u\left(w_{0}\right) d w_{0} \\
& =\frac{1}{2 \pi i} \int_{\mathcal{C}} K\left(w_{0}-z_{0}\right)\left(-\partial_{w_{0}}\right)^{n} u\left(w_{0}\right) d w_{0}+\tilde{u}_{n}(z)
\end{aligned}
$$

where

$$
\tilde{u}_{n}(z)=\left.\sum_{\imath=0}^{n-1}\left(\partial_{w_{0}}\right)^{n-1-\imath} K\left(w_{0}-z_{0}\right)\left(-\partial_{w_{0}}\right)^{\imath} u\left(w_{0}\right)\right|_{w_{0} \in \partial \mathcal{C}}
$$

and $\partial \mathcal{C}$ means the boundary of $\mathcal{C}$, that is, the starting point and the endpoint. Since $A(\lambda)=\sum_{n=0}^{+\infty} a_{n} \lambda^{n}$ with $\left.\left|a_{n}\right| \leq A B^{n} / \Gamma(n(\gamma+1) / \gamma)+1\right)$, by putting $\tilde{u}(z)=\sum_{n=0}^{+\infty} a_{n} \tilde{u}_{n}(z)$, we have (3.13).

(ii) We have

$$
\partial_{z_{0}}^{n}(K u)\left(z_{0}\right)=\frac{(-1)^{n}}{2 \pi i} \int_{\mathcal{C}} K^{(n)}\left(w_{0}-z_{0}\right) u\left(w_{0}\right) d w_{0} .
$$

Here we choose path $\mathcal{C}$ as follows: let $\hat{z}_{0}=\sqrt{a^{2}-y_{0}^{2}}+i y_{0}$ and $\mathcal{C}=\mathcal{C}_{0}+\mathcal{C}_{1}(\epsilon)+$ $\mathcal{C}_{2}(\epsilon)-\mathcal{C}_{1}(\epsilon)-\mathcal{C}_{0}$

$$
\begin{aligned}
\mathcal{C}_{0} & =\left\{w_{0}=s \hat{z}_{0}+(1-s) a ; 0 \leq s \leq 1\right\} \\
\mathcal{C}_{1}(\epsilon) & =\left\{w_{0}=s\left(z_{0}+\epsilon\right)+(1-s) \hat{z}_{0} ; 0 \leq s \leq 1\right\} \\
\mathcal{C}_{2}(\epsilon) & =\left\{w_{0}=z_{0}+\epsilon e^{2 \pi \imath s} ; 0 \leq s \leq 1\right\}
\end{aligned}
$$

where $\epsilon>0$ is sufficiently small constant.

We have $0 \leq \arg \left(w_{0}-z_{0}\right) \leq 2 \pi$ for $w_{0} \in \mathcal{C}_{1}(\epsilon) \cup \mathcal{C}_{2}(\epsilon)$. Hence $K^{(n)}\left(w_{0}-z_{0}\right)$ is bounded on $\mathcal{C}_{1}(\epsilon) \cup \mathcal{C}_{2}(\epsilon)$ by Lemma 3.4-(ii). By letting $\epsilon \rightarrow 0$,

$$
\begin{aligned}
& \partial_{z_{0}}^{n}(K u)\left(z_{0}\right)=\left(K_{0}^{n} u\right)\left(z_{0}\right)+\left(K_{1}^{n} u\right)\left(z_{0}\right) \\
& \left(K_{0}^{n} u\right)\left(z_{0}\right)=\frac{(-1)^{n}}{2 \pi i}\left(\int_{\mathcal{C}_{0}}+\int_{-\mathcal{C}_{0}}\right) K^{(n)}\left(w_{0}-z_{0}\right) u\left(w_{0}\right) d w_{0} . \\
& \left(K_{1}^{n} u\right)\left(z_{0}\right)=\frac{(-1)^{n}}{2 \pi i} \int_{\mathcal{C}_{1}(0)}\left\{K^{(n)}\left(w_{0}-z_{0}\right)-K^{(n)}\left(\left(w_{0}-z_{0}\right) e^{2 \pi \imath}\right)\right\} u\left(w_{0}\right) d w_{0} .
\end{aligned}
$$




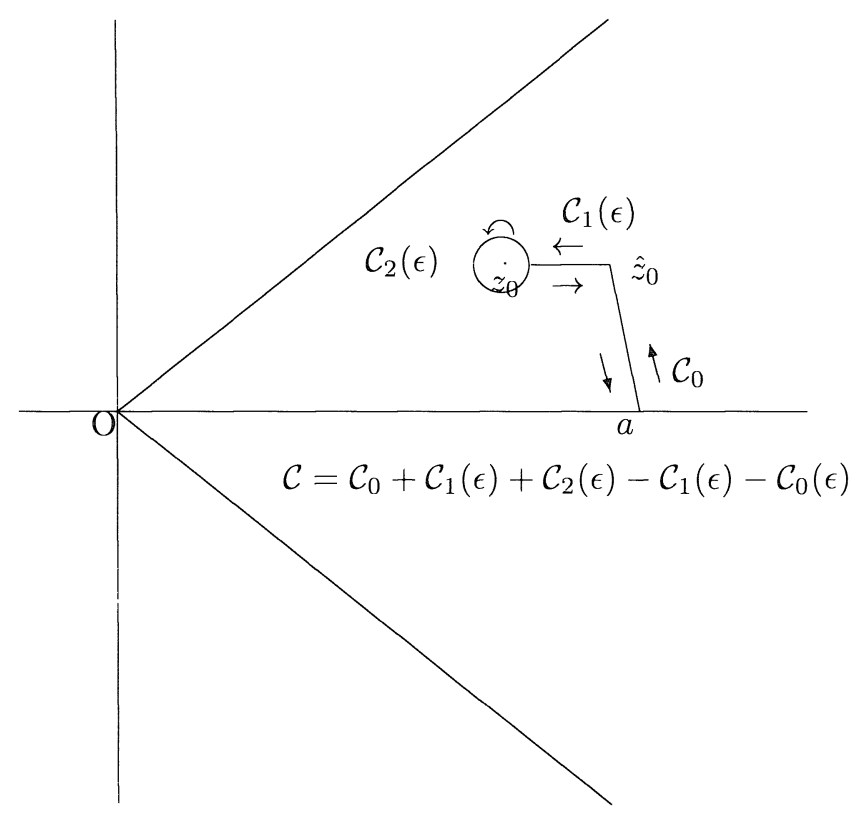

Figure 2. Path $\mathcal{C}$

We have $\left|\left(K_{0}^{n} u\right)\left(z_{0}\right)\right| \leq A D^{n} n$ ! for $z_{0} \in V_{0}\left(\theta_{0}\right)$. As for $\left(K_{1}^{n} u\right)\left(z_{0}\right)$ it follows from Lemma 3.4-(iii) that for small $\varepsilon>0$

$$
\begin{aligned}
& \left|\left(K_{1}^{n} u\right)\left(z_{0}\right)\right| \\
\leq & A_{\varepsilon} D_{0}^{n} \Gamma\left((n+1)\left(\frac{\gamma+1}{\gamma}\right)\right) \int_{\mathcal{C}_{1}(0)} \exp \left(-c\left|w_{0}-z_{0}\right|^{-\gamma}+\varepsilon\left|w_{0}\right|^{-\gamma}\right)\left|d w_{0}\right| \\
\leq & A_{\varepsilon}^{\prime} D_{1}^{n} \Gamma\left((n+1)\left(\frac{\gamma+1}{\gamma}\right)\right) .
\end{aligned}
$$

Hence $\left|\partial_{z_{0}}^{n}(K u)\left(z_{0}\right)\right| \leq A_{1} D_{2}^{n} \Gamma\left((n+1)\left(\frac{\gamma+1}{\gamma}\right)\right)$ for $z_{0} \in V_{0}\left(\theta_{0}\right)$. Thus $\partial_{z_{0}}^{n}(K u)\left(z_{0}\right)$ is bounded in $V_{0}\left(\theta_{0}\right)$ for any $n \in \mathbb{N}$ and has Gevrey type estimate, which means that $\lim _{z_{0} \rightarrow 0}\left(K^{n} u\right)\left(z_{0}\right)$ exists in $V_{0}\left(\theta_{0}\right)$ and $(K u)\left(z_{0}\right) \in A s y_{\{\gamma\}}\left(V_{0}\left(\theta_{0}\right)\right)$.

Let $c>0$ and $A_{c}(\lambda)=A(c \lambda)$ and define

$$
K_{c}(t)=\frac{1}{2 \pi i} \int_{0}^{\infty e^{i \varphi}} \frac{\exp (\lambda t)}{A_{c}(\lambda)} d \lambda .
$$

Then the similar results are valid if we replace $A(\lambda)$ by $A_{c}(\lambda)$ and $K(\lambda)$ by $K_{c}(\lambda)$ respectively. We note that $A_{c}(\lambda)=\sum_{n=0}^{+\infty} a_{n}(c) \lambda^{n}$ with $a_{n}(c)=c^{n} a_{n}$ 
and $\left|a_{n}(c)\right| \leq A(c B)^{n} / \Gamma(n(\gamma+1) / \gamma+1)$, where the constants $A$ and $B$ are those in (3.7)

Proof of Theorem 3.1. If $\gamma=+\infty,(\gamma+1) / \gamma=1$ and it is easy to show that $u(z)$ is holomorphic at $z=0$. Suppose that $0<\gamma<+\infty$ and $u\left(z_{0}\right)$ satisfies (3.1):

$$
\left|\left(\frac{\partial}{\partial z_{0}}\right)^{n} u(z)\right| \leq M_{\varepsilon} \exp \left(\varepsilon\left|z_{0}\right|^{-\gamma}\right) C^{n} \Gamma\left(n\left(\frac{\gamma+1}{\gamma}\right)+1\right) \text { for } z \in U\left(\theta^{\prime}\right)
$$

holds for all $n \in \mathbb{N}$. Let $A_{c}(\lambda)=A(c \lambda)$ and choose $c>0$ so that $c C B<1$. Let $0<\theta^{\prime}<\min \{\pi / 2, \theta\}$ and $0<\theta_{0}<\theta^{\prime}$. Then, by the above estimate of $\partial_{0}^{n} u(z), v\left(z_{0}\right)=A_{c}\left(-\partial_{z_{0}}\right) u\left(z_{0}\right)$ converges and $v\left(z_{0}\right) \in U_{(\gamma)}\left(\theta^{\prime}\right)$. It follows from Proposition 3.5 that

$$
\begin{aligned}
-u\left(z_{0}\right) & =A_{c}\left(-\partial_{z_{0}}\right)\left(K_{c} u\right)\left(z_{0}\right) \\
& =\frac{1}{2 \pi i} \int_{\mathcal{C}} K_{c}\left(w_{0}-z_{0}\right) A_{c}\left(-\partial_{w_{0}}\right) u\left(w_{0}\right) d w_{0}+\tilde{u}\left(z_{0}\right) \\
& =\left(K_{c} v\right)\left(z_{0}\right)+\tilde{u}\left(z_{0}\right) .
\end{aligned}
$$

Since $\left(K_{c} v\right)\left(z_{0}\right) \in A s y_{\{\gamma\}}\left(V_{0}\left(\theta_{0}\right)\right)$ and $\tilde{u}\left(z_{0}\right)$ is holomorphic at the origin, we have $u\left(z_{0}\right) \in A s y_{\{\gamma\}}\left(V_{0}\left(\theta_{0}\right)\right)$. If $\theta>\pi / 2$, we have the conclusion of Theorem 3.1 by the rotation with respect to $z_{0}$.

\section{References}

[1] Hamada, Y., Leray, J. et Wagschal, C., Système d'équation aux derivées partielles à caractéristic multiples; problème de Cauchy ramifié; hyperbolicité partielle, J. Math. Pures Appl., 55 (1976), 297-352.

[2] Komatsu, H., Ultradistribution, I, Structure theorems and a characterization, J. Fac.

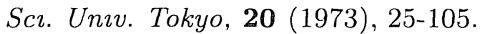

[3] - Irregularity of characteristic elements and construction of null solutions, J. Fac. Scı. Unıv. Tokyo, 23 (1976), 297-342.

[4] Ōuchi, S., Index, localization and classification of characteristic surfaces for linear partial differential operators, Proc. Japan Acad., 60 (1984), 189-192.

[5] - An integral representation of singular solutions and removable singularities to linear partial differential equations, Publ. RIMS, Kyoto Unvv., 26 (1990), 735-783.

[6] - Singular solutions with asymptotic expansion of linear partial differential equations in the complex domain, Publ. RIMS, Kyoto Unvv., 34 (1998), 291-311.

[7] _ - Growth property and slowly increasing behavior of singular solutions of linear partial differential equations in the complex domain, to appear in J. Math. Soc. Japan.

[8] Wagschal, C., Problème de Cauchy analytique à données méromorphes, J. Math. Pures Appl., 51 (1972), 375-397. 